\title{
Mid-Infrared Spectra of Be Stars
}

\author{
S. A. Rinehart, J. R. Houck, \& J. D. Smith \\ Cornell University \\ rinehart@tristan.tn.cornell.edu, jrh13@cornell.edu, \\ jdsmith@astrosun.tn. cornell.edu
}

\begin{abstract}
We present the first medium-resolution $(R \sim 600)$ mid-infrared $(8-13.3 \mu \mathrm{m})$ spectra of 11 Be stars. A large number of lines are observed and identified in these spectra, including, as an example, 39 hydrogen recombination lines in the spectrum of $\gamma$ Cas. In the majority of our spectra, all of the observed lines are attributable to hydrogen recombination. Two of the sources, $\beta$ Lyr and MWC 349 also show emission from other species. Both of these objects show evidence of [Ne II] emission, and $\beta$ Lyr also shows evidence of He I emission. We tabulate the effective line strength and line widths for the observed lines, and briefly discuss the physical implications of the observed line series. We also use a simple model of free-free emission to characterize the disks around these sources.
\end{abstract}

Subject headings: Infrared Radiation — Stars: Emission-line, Be

\section{Introduction}

Be stars are defined as B stars which display optical emission in one or more Balmer lines of hydrogen, or which have displayed such emission at some point in the past. There are also several subdivisions of Be stars, including the "classical" Be stars and Herbig Be stars. Herbig Ae/Be stars are considered the intermediate mass $\left(5-20 M_{\odot}\right)$ counterparts to $\mathrm{T}$ Tauri stars; they are pre-main sequence stars surrounded by an optically thick, dusty disk remaining from proto-stellar collapse. From this disk arises forbidden line emission and $\mathrm{H} \alpha$ emission. Classical Be stars, on the other hand, are intermediate mass main-sequence objects, believed to have an extended optically thin gaseous envelope. While these two types of objects share similar nomenclature, they have little in common outside of their masses.

The sources presented in this paper are all classical Be stars, and henceforth the term Be star refers to classical Be stars. Classical Be stars are also divided into two categories, "non-shell",

\footnotetext{
${ }^{1}$ Now at Queen Mary \& Westfield College, Mile End Road, London E1 4NS, United Kingdom, email at S.Rinehart@qmw.ac.uk
} 
or "normal" stars, and "shell" stars. Shell stars display broad emission wings in one or more of the hydrogen lines and narrow absorption bands for ionized metals (Slettebak 1988), features not observed in non-shell Be stars. Several suggestions about the relation between shell stars and normal Be stars have been made: this relation is complicated by the fact that several stars, including $\gamma$ Cas and Pleione (HR 1180), have been observed to change from "normal" spectra to "shell" spectra (Doazan \& Thomas 1982). Hanuschik (1996) explains shell stars as Be stars which are observed equator-on, with the transition between normal and shell spectra explained by density variations in a concave equatorial disk viewed at a small inclination angle $\left(\sim 15^{\circ}\right)$. Alternatively, it has been suggested that the shell phase is a common evolutionary phase of Be stars (Slettebak, et al. 1992), involving the formation of a gas shell outside the stellar envelope. The formation of the gas shell can explain the transition from normal to shell spectra, while the dissipation of the gas shell causes the transition from shell to normal spectra.

Optical spectroscopy has raised a number of questions about Be stars. Individual spectral lines are found to be double peaked, with a ratio of the violet peak flux to the red peak flux (the $V / R$ ratio) which differs from unity. Further, this ratio and the line strengths of the hydrogen Balmer lines have been found to be time-variable in many Be stars (Telting et al 1993).

Near-infrared (NIR) spectroscopy has added to this puzzle. Variability of line strengths and $\mathrm{V} / \mathrm{R}$ ratios of the double-peaked lines mirror observations in the optical (Hamann \& Simon 1989). In addition, a number of the hydrogen recombination lines which appear in the NIR have anomalously high line strengths, and the NIR continuum has been observed to vary with time (Dougherty \& Taylor 1994).

Ultraviolet observations complicate the problem further. The strong lines observed in the UV should arise from a fast, low-density wind (Smith 1995). This is at odds with the optical and NIR observations, which require a relatively dense gas to produce the observed features. A possible explanation for this discrepancy is discussed below.

X-ray observations have shown several Be stars to be X-ray binaries. $\gamma$ Cas, the brightest of the northern hemisphere Be stars has a compact companion, most likely a white dwarf (Haberl 1995). Other Be stars are also in binary systems; a large number of Be stars, including many of the sources presented in this sample, are spectroscopic binaries, including $\beta$ Lyr, $\zeta$ Tau, and $\phi$ Per (Jared et al. 1989).

Of all of the wavelength regions, the mid-infrared has been the least explored. Photometry by Gehrz et al (1974) from 2.3 to $19.5 \mu \mathrm{m}$ showed that the spectral energy distribution of the IR excess was consistent with free-free emission from a warm disk-shaped envelope. It has also been suggested (when discussing NIR observations) that studies of infrared line emission can be very useful in probing the stellar envelope of Be stars, as the emission is highly dependent upon the temperature and density structure of the envelope (Marlborough et al. 1997). This is one of the motivations for the study introduced here.

Explaining the multitude of observations by modelling is a daunting task. The most common 
model used to explain the observations is the axisymmetric equatorial disk model of Poeckert \& Marlborough (1978). A slow, high-density wind at the equator creates a thick envelope which produces the observed optical and NIR emission, while the UV emission arises from a fast, tenuous wind originating at the poles of the star (Lamers \& Waters 1987). The presence of an equatorial disk has been supported by interferometric and polarimetric observations (Quirrenbach et al. 1997, Stee et al. 1995), but these models are incomplete, as they still cannot explain some Be star phenomenae, such as the observed $\mathrm{V} / \mathrm{R}$ variability.

A more complete discussion of Be stars can be found in several excellent review articles, including Slettebak (1988) and Dachs (1986).

In this paper, we present the first high-sensitivity, medium resolution $(R \sim 600)$ mid-infrared $(8-13.3 \mu \mathrm{m})$ spectra of be stars. In $\S 2$, we discuss the observations and data reduction techniques. In $\S 3$, we present the observed spectra, and briefly mention characteristics of particular sources. In $\S 4$, we discuss using free-free emission from the stellar envelope to model the MIR continuum. Finally, in $\S 5$, we briefly discuss some of the implications of these observations.

\section{Observations and Data Reduction}

The observations presented here were taken using SCORE (van Cleve et al. 1998) at the Cassegrain focus of the Hale 5-m telescope. ${ }^{2}$ SCORE is a novel medium resolution mid-infrared spectrograph built originally as a proof of concept of the Infrared Spectrograph (IRS) short-hi module for SIRTF. It uses cross-dispersion to obtain a $7.5-15 \mu \mathrm{m}$ spectrum in a single exposure. The obtained spectrum has a resolution of $\sim 600$, with a $1^{\prime \prime} \times 2^{\prime \prime}$ slit. This short slit length is ideal for sources which are unresolved in the MIR at Palomar. SCORE also makes use of a second MIR array for slit-viewing, which both simplifies source acquisition during observing and provides information for photometric calibration (see discussion below). A listing of all of the observations is presented in Table 1. All of these observations were made using standard beam-switched chopping and nodding techniques. Typical integration times ranged from 240 to 1090 seconds, and the entire $\mathrm{N}$-band was observed at a single setting for each object. The $1 \sigma$ sensitivity at $11.0 \mu \mathrm{m}$ in 100 seconds of integration for our instrument is roughly $90 \mathrm{mJy}$. The MIR seeing for these observations was typically $1^{\prime \prime}$.

The individual integrations of each object were stacked, and the N-band spectra were extracted using SCOREX, extraction software designed to optimally extract the spectrum from the crossdispersed format of SCORE (Smith et al. 1998). These spectra were then calibrated using observed spectra of standard stars (Cohen, et al. 1992) to properly remove spectral features from the sky background. The observed spectra from these standard stars were taken at nearly the same airmass

\footnotetext{
${ }^{2}$ Observations at the Palomar Observatory were made as part of a continuing collaborative agreement between the California Institute of Technology, the Jet Propulsion Laboratory, and Cornell University.
} 
as our observed objects, as even relatively small differences in airmass result in different strengths of atmospheric features, leading to inaccurate subtraction of background features from our calibrated spectra.

Absolute photometry for the spectra was found by using the slit-viewer images of SCORE. Since a small chop amplitude $\left(\sim 5^{\prime \prime}\right)$ was used for these observations, the chopped images appear in the field-of-view of the slit-viewer. We calculate the slit-throughput by comparing the flux incident on the slit-viewer focal plane with the total flux found in the observed spectrum; mathematically,

$$
A=\frac{F_{\nu}(\text { Slitviewer })}{\int F_{\nu}(\lambda) g(\lambda) \mathrm{d} \lambda}
$$

where $A$ is the renormalization factor, $F_{\nu}(\lambda)$ is the flux as a function of wavelength on the spectrograph, $g(\lambda)$ is the slit viewer filter passband function, and $F_{\nu}$ (Slitviewer) is the total flux incident on the slit-view focal plane array. The renormalization factor $A$ should account for the slit throughput. To provide the absolute calibration, we use the same procedure for our calibrator star, deriving another normalization factor, $A_{\text {calib }}$. By multiplying the observed, calibrated spectrum of our source

by $\frac{A}{A_{\text {calib }}}$ we are able to normalize the observed spectrum to have the correct amount of flux, without introducing errors from instrumental effects. The absolute normalization of the spectra is uncertain to roughly $5 \%$, due primarily to the uncertainty in the slit throughput efficiency.

\section{Observed Spectra of Be stars}

In Figure 1, we present the reduced spectra of our objects. These spectra clearly show the presence of a large number of spectral lines. Further, in most of the spectra, there are a number of lines which are weak relative to the strong MIR continuum. Since these weak lines appear in several different spectra, but not in calibration star spectra, we conclude that they are not artifacts. Further, the clear presence of multiple lines in each series implies the presence of other lines within the series. For instance, the $\mathrm{H} 29 \rightarrow 10$ is weak in the spectrum of $\gamma$ Cas, but the presence of other transitions, (including the $\mathrm{H} 29 \rightarrow 9$ transition in this case) support the identification of this weak line, since they arise from a common excited state. Similar support can be found for nearly all of the weak observed lines. Finally, because all of the lines occur at wavelengths attributable to hydrogen recombination (for most of our objects, see below), there is strong evidence to support the pure-hydrogen recombination spectra.

There is also another test for the presence of these weak lines. Nine of the 11 spectra display similar strong hydrogen line emission (all but $\beta$ Lyr and MWC 349), but a number of these spectra have low signal to noise. These nine sources will hereafter be referred to as the hydrogen spectra Be stars (or HS stars). If we suppose that all of the lines observed in the high signal to noise spectra (such as $\gamma$ Cas and $\zeta$ Tau) are present in the spectra of all HS sources, we can coadd the spectra from all nine of these objects. If these lines are, in fact, present in a majority of the sources, we 
expect the weak lines to be accentuated. If, on the other hand, these weak lines are present in only a few spectra, we expect them to be diluted (relative to their appearance in the individual spectra). This coadded spectrum is shown in Figure 2, with the line identifications marked at the top of the plot. Examination of this spectrum shows a large number of lines, including a number which are only observed as very faint peaks in the spectra of our bright sources. This supports the idea that all of the observed hydrogen transitions are present in the majority of the HS sources.

In Table 2, we list all of the observed lines, their line widths and effective line strengths, and the hydrogen recombination transition responsible for their production, in order of decreasing wavelength. This table includes the data for all of the HS sources (which excludes $\beta$ Lyr and MWC 349). The data from the two peculiar objects ( $\beta$ Lyr and MWC 349) is presented in Table 3, because of the clear differences between their spectra and the spectra of the majority of our sources. Note that in Table 2, every observed line is attributable to hydrogen recombination. We examined the spectra for the presence of line species other than hydrogen, in particular for the presence of He I or He II, since these species can produce emission lines coincident with hydrogen lines. He I is easily identified by the presence of the $10.88 \mu \mathrm{m}$ He I $3 \mathrm{~S}-3 \mathrm{P}_{o}$ transition, while He II can be identified by the presence of many additional hydrogen-like transitions. In the nine HS spectra, there is no evidence for the He I $10.88 \mu \mathrm{m}$ transition or for the numerous He II transitions which would be present if the species accounted for any of the observed emssion.

In $\beta$ Lyr, however, we observed the He I 3S-3P $\mathrm{P}_{o}$ transition at $10.88 \mu \mathrm{m}$. Since Helium recombinations occur at the same wavelength as hydrogen recombinations (for instance, both $\mathrm{H} 9 \rightarrow 7$ and He I $9 \rightarrow 7$ occur at $11.3 \mu \mathrm{m}$ ) the presence of this line implies that the observed lines are not necessarily purely due to hydrogen, as is true for most of our other sources. The possible overlap of hydrogen and helium recombination line makes exact identification of these lines problematic. We also find evidence for the [Ne II] $12.8 \mu \mathrm{m}$ transition, a line also observed in the spectrum of MWC 349. MWC 349 showed no evidence for He I or He II emission, and only showed a few weak hydrogen transitions (with the exception of the $\mathrm{H} 7 \rightarrow 6$ transition which was quite strong). We will return to a discussion of the observed spectral features of these objects later in this section.

\subsection{Hydrogen Spectra Be Stars}

While the nine HS sources presented here have similar MIR spectra, they are significantly different types of objects in several ways. Seven of the sources are classified as shell stars. Five of our sources are spectroscopic binaries, and three of the sources are X-ray binaries. Further, while most Be stars do not display radio emission, four of the sources presented here are relatively bright radio sources. The characteristics of the individual objects are tabulated in Table 4. For completeness, some of the characteristics of the two peculiar sources are also listed on this table.

The large differences in the properties of the individual objects leads us to look for correlations. Are any of these individual properties related to the MIR observations presented here? We looked 
at correlation plots for each of the different categories listed in Table 4. An example of one of these correlation plots is shown in Figure 3. We have plotted the ratio of the $\mathrm{H} 9 \rightarrow 7$ to $\mathrm{H} 7 \rightarrow 6$ transition versus the ratio of the $\mathrm{H} 10 \rightarrow 7$ to $\mathrm{H} 7 \rightarrow 6$ transitions in this figure, with the XRB plotted as + symbols, spectroscopic binaries as open triangles, and single stars as open diamonds. We have examined a number of similar correlation plots, looking for evidence that our MIR spectra are effected by each of these properties, but have found no such evidence. However, given the small statistics (only nine sources), this lack of detection is not surprising, and only by building a more significant set of data will such correlation tests be valuable for understanding the effects of these peculiarities on our MIR spectra.

The presence of so many high-level hydrogen transitions provides valuable insight into the origin of line emission. The line strengths are inconsistent with optically thin line emission (Hummer \& Storey 1987), and therefore must originate at optical depths of $\sim 1$. The optically thick emission will simply be the product of the Planck function, the line width, and the surface area of the emission region. Since the Planck function of a gas with temperatures consistent with the production of such high $\mathrm{H}$ recombination series decreases with wavelength and our observations show no clear trend in line width with wavelength, the surface area must be increasing with wavelength. ISO observations have shown that the line width actually decreases with wavelength (Hony et al. 1999), strengthening this statement. This implies a density gradient, in order to balance line ratios; if the density is uniform, then the low-level transitions will become optically thick much more quickly than their high-level counterparts, giving them an effective emission surface significantly larger than the high-level lines, and overcompensating for the decreasing strength with wavelength. This was previously noted from NIR observations of $\gamma$ Cas (Hamann \& Simon 1987). These authors concluded that gradients as small as $\mathrm{r}^{-2}$ were sufficient to explain their observed line ratios.

\subsection{Peculiar Be Stars}

$\beta$ Lyr and MWC 349 display spectra which are significantly different from our HS sources, and from each other. Each of these sources is unique, and it is therefore not surprising that their MIR spectra should be peculiar as well. We briefly discuss the peculiar aspects of the MIR spectra of these objects here, and present some possible explanations for these peculiarities.

$\beta$ Lyr is a known spectroscopic and eclipsing binary. The two members of this binary are $\mathrm{B}$ stars, with masses of $M_{1} \approx 13 M_{\odot}$ and $M_{2} \approx 3 M_{\odot}$ (Harmanec \& Scholz 1993). The two stars are in close orbit, with the mass-loss from the less-massive star filling its Roche lobe, overflowing to form an accretion disk around the more massive star (De Greve \& Linnell 1994). This situation is quite different from the disk-like stellar envelopes around our HS Be stars, which occupy a comparatively small volume.

The accretion process can also possibly explain observed He I emission by invoking convective dredging in the donor star, thus bringing helium into the Roche lobe and the accretion disk. The 
presence of the He recombination lines in this spectrum, when compared to the HS sources is most easly explained by either a higher He abundance in the circumstellar disk or a higher level of excitation. If we compare $\beta$ Lyr to $\gamma$ Cas, however, we see that $\gamma$ Cas is significantly hotter than $\beta$ Lyr $\left(T_{\beta L y r}<20000 \mathrm{~K}\right)$. Since no He emission is observed in $\gamma$ Cas, whose disk gas is at higher excitation, we conclude that the observed He emission in $\beta$ Lyr is due to a greater He abundance.

The biggest question raised from examination of the MIR spectrum of $\beta$ Lyr is the cause of [Ne II] emission. While a great number of emission lines have been observed in the optical and NIR spectra of $\beta$ Lyr, there has been no evidence for forbidden emission in any of these spectra (Johnson 1978), or for mid-infrared emission from forbidden lines in our spectra ([Ar III] at $9.0 \mu \mathrm{m}$, [S IV] at $10.5 \mu \mathrm{m})$. Why should [Ne II] emission, an no other forbidden emission, be observed? The two properties which should be examined in particular are the ratio of the observed to critical density $\left(n_{e} / n_{\text {crit }}\right)$ for [Ne II] emission and the energy required to form Ne II. Upon examination, we find that both of these characteristics of $\mathrm{Ne}$ are favorable for the observed forbidden emission. [Ne II] has a very high critical density $\left(10^{5} \mathrm{~cm}^{-3}\right)$, higher than any other astronomically strong MIR forbidden transition. Further, it has a relatively low ionization potential $(21.5 \mathrm{eV})$, making it possible to ionize a large enough fraction of the Neon gas by the hot B stars to create the observed emission.

Using the observed strength of the [Ne II] line, the distance to $\beta$ Lyr $(270 \mathrm{pc}$ ) from the Hipparchos catalog (1997), and making a few assumptions about the emitting gas (e.g. $n_{e}=$ $n_{\text {crit }}, T_{e}=10^{4} \mathrm{~K}$, solar abundances), we can calculate an approximate mass of emitting gas. This calculation gives a mass of the emitting gas of $M_{t o t} \approx 10^{-5} M_{\odot}$. This compares favorably with calculations of the mass-transfer rate from the "donor" star to the "accretor" star, if we assume that some small fraction of mass-transfer escapes into an extended gas shell. Such calculations, assuming complete mass conservation between the two stars, give values of $\dot{M} \approx 2 \times 10^{-5} \mathrm{M}_{\odot} \mathrm{yr}^{-a}$ (Hubeny et al. 1994). If we assume that the gas is fully ionized, we can estimate an emission volume for the [Ne II] line. We assume, for this approximation, that the emission volume is spherical, and find that the emitting region should be of order 100 AU across. For comparison, the separation of the two components of the binary is only of order 20 AU. From these estimates, we suggest that the forbidden line emission does not arise in the Roche lobe or accretion disk, but must come from a much more extended gas shell around the binary. This could also explain the difference in the observed hydrogen spectra of this source, relative to our HS sources. The extended, low density outer gas shell will produce optically thin line emission from hydrogen, while the disk produces optically thick line emission. The contribution from the optically thin emission will alter the hydrogen line ratios, explaining why the line ratios for hydrogen emission from $\beta$ Lyr are not $\sim 1$ (as for our HS sources).

MWC 349 has the largest MIR to optical flux ratio of all of our sources, with this ratio more than 100 times larger than our other sources. This could be due to several different phenomena, including a much cooler source or much more circumstellar mass (including, perhaps, a large amount of dust, leading to severe reddening). Independent of the cause, the red color of MWC 349, 
compared to our HS sources, implies that the nature of this object or its environment may be quite different from classical Be stars. Observations of MWC 349 in the submillimeter detected $\mathrm{H} \alpha$ transitions which were greatly amplified; MWC 349 was the first source observed with hydrogen laser emission (Martin-Pintado et al. 1989). Further, recent ISO observations have detected more than 160 emission lines between 2.4 and $190 \mu \mathrm{m}$, including every hydrogen $\alpha$ transition (H5 $\rightarrow 4$ to $\mathrm{H} 16 \rightarrow 15$ ) in this range (Thum et al. 1998), showing the presence of infrared lasers from the large amplification of the $\mathrm{H} \alpha$ lines. Further, lasing/masing has been observed from a number of the hydrogen $\beta$ lines. It is thought that the conditions which allow the observed masing/lasing from MWC 349 include the high temperature of (and corresponding large ultraviolet flux from) the central star, a dense, massive, neutral disk (much more massive than the disks in most Be stars), and the coincidental edge-on view we have of the disk (Strelnitski et al. 1996).

A significant amount of forbidden line emission in the optical has been observed in MWC 349 (Brugel \& Wallerstein 1979, Andrillat \& Swings 1976, Allen \& Swings 1976)). We have also detected forbidden emission, in the form of the [Ne II] $12.81 \mu \mathrm{m}$ line. It is unlikely that this forbidden emission arises from the same region as the hydrogen emission, however. Recent work by Them \& Greve (1997) used the observed Paschen decrement to estimate the electron density in the disk at $n_{e}=10^{8}$, over one hundred times greater than the critical density for [Ne II] forbidden emission. The forbidden emission more likely arises in an extended gas component around the star. Such an extended component has been observed via radio measurements and has been associated with a slow $\left(50 \mathrm{~km} \mathrm{~s}^{-1}\right.$ ) gaseous outflow. Cohen et al. (1985), using observations from the VLA, found that the radio observations were well-matched by a spherical $1 / r^{2}$ wind model, with mass-loss rates of order $10^{-5} \mathrm{M}_{\odot} \mathrm{yr}^{-1}$ and a temperature of roughly $9000 \mathrm{~K}$.

Using the same technique as described for $\beta$ Lyr, we estimate the mass of the emitting gas from the forbidden emission. We use the same assumptions as in the case of $\beta$ Lyr, and estimate a distance to the source of $400 \mathrm{pc}$ (Thompson et al. 1977). From these, we find a mass of the emitting gas of $M_{t o t} \approx 10^{-2} M_{\odot}$. The mass of the disk should be substantially larger than this, up to several solar masses (Thompson et al., Thum \& Greve 1997). However, the forbidden emission we observe will only come from the regions around the star where the density is relatively low, and therefore will only arise from a small fraction of the disk, since the majority of the disk has a high $\left(\sim 10^{8} \mathrm{~cm}^{-3}\right)$ density (Thum et al. 1998).

\section{Continuum Emission}

The majority of photospheric radiation from hot stars is at short wavelengths, in the optical or ultraviolet (an $16000 \mathrm{~K}$ star has a peak flux at $\sim 1800 \AA$, well into the UV). In the MIR, photospheric emission is well inside the Raleigh-Jeans tail of the blackbody distribution, producing relatively weak emission in this wavelength range. Classical Be stars have been long known to produce large IR excesses, and a common explanation for this is emission via free-free processes. 
Free-free radiation from ionized gas is a powerful source of emission at long wavelengths. Typically, such emission has been used to explain radio emission from cool astronomical sources (Panagia \& Felli 1975), but it can also explain the infrared excesses observed from many sources. Be stars have well-known IR excess, and free-free emission from the extended stellar envelope provides a convenient explanation for this excess.

Gehrz et al. (1974) used models of optically thin and optically thick free-free emission to match infrared photometry of Be stars from $2.3 \mu \mathrm{m}$ to $19.5 \mu \mathrm{m}$. Waters et al (1984) successfully modeled IRAS Be star observations with an optically thick free-free emission. We have used models of freefree emission to fit our MIR continuum observations, finding values for the shell radius $\left(R_{s h}\right)$, the electron density $\left(n_{e}\right)$, and the shell temperature $\left(T_{s h}\right)$.

\subsection{Calculations of Free-Free Emission}

Three emission processes are included in our model of the MIR continuum: emission from the photosphere of the star, optically thin free-free emission, and optically thick free-free emission.

Because neither of the free-free processes are very effective at short wavelengths, we can constrain the stellar parameters without having to consider the effects of the shell. In the infrared, the photospheric spectrum is essentially a blackbody produced at the photospheric temperature, so the Planck function describes this emission. Initial values for the temperature $\left(T_{\star}\right)$ and radii $\left(R_{\star}\right)$ of our sources were approximated from their respective spectral types (Waters et al. 1987), and we then adjusted the values of both $T_{\star}$ and $R_{\star}$ to agree with J-band $(1.25 \mu \mathrm{m})$ and K-band $(2.20 \mu \mathrm{m})$ photometry (Gezari et al. 1993). Distances to the stars were obtained from the Hipparchos Catalog (1997). All of these parameters, including the J-band and K-band fluxes, are listed in Table 5.

To fit the observed MIR continuum, we require several assumptions. First, we assume that the MIR emission arises from an extended stellar envelope around the star. Further, it is assumed that this envelope is flattened into an oblate spheroidal disk, with a semi-minor axis $\approx 1 / 10 R_{s h}$ where $R_{s h}$ is the semi-major axis of the spheroid. Further, we assume that the stellar envelope has both uniform density and uniform temperature. These assumptions are clearly not correct, but greatly simplify calculations without introducing significant amounts of error. Finally, we assume that we are looking into the disk-like envelope edge-on. This last assumption is supported by two facts: the large values of $v \sin i$ for these sources, and the central line absorption of shell spectra (seven of our nine HS sources are shell stars, see Table 4).

The MIR emission from the stellar photosphere is calculated using the Planck function (making use of $R_{\star}, T_{\star}$, and $D$ ), as described above, but is corrected by applying an extinction factor in the form of $e^{-\tau\left(\rho=R_{s h}\right)}$. This will account for free-free absorption of photospheric radiation as a function of wavelength. Thus, we write 


$$
F_{\lambda}^{\star}=37469.4\left[e^{\left(1.44 \frac{1 \mu \mathrm{m}}{\lambda} \frac{10^{4}}{T_{\star}}\right)}-1\right]^{-1}\left(\frac{1 \mu \mathrm{m}}{\lambda}\right)^{5}\left(\frac{R_{\star}}{D}\right)^{2} e^{-\tau\left(\rho=R_{s h}\right)}
$$

Next, we include the effect of optically thick free-free emission. The optically thick emission will be characterized by the Planck function at the shell temperature $\left(T_{s h}\right)$. This emission will arise from an area of the disk which is defined by the surface a depth $\rho$ into the disk, where the optical depth is

$$
\tau^{f f}=\alpha_{\nu}^{f f} \rho=1.4 \times 10^{-3}\left(\frac{n_{e}}{10^{11}}\right)^{2}\left(\frac{10^{4}}{T_{s h}}\right)^{0.5}\left(\frac{\lambda}{\mu \mathrm{m}}\right)^{3}\left(1-e^{-1.44 \frac{\mu \mathrm{m}}{\lambda} \frac{10^{4}}{T_{s h}}}\right)\left(\frac{\rho}{10^{12}}\right)=1
$$

This cuts off the optically thick emission at short wavelengths, as the absorption coefficient becomes very small and the entire disk becomes optically thin. Mathematically,

$$
F_{\lambda}^{f f-t h i c k}=37469.4\left[e^{\left(1.44 \frac{1 \mu \mathrm{m}}{\lambda} \frac{10^{4}}{T_{s h}}\right)}-1\right]^{-1}\left(\frac{1 \mu \mathrm{m}}{\lambda}\right)^{5}\left(\frac{R_{s h}}{D}\right)^{2} x(\tau)
$$

where $x(\tau)$ is the ratio of the area of the optically thick emission to the maximum area of optically thick emission.

Finally, we include the effect of the optically thin region of the emission disk. This can be accounted for to first order by integrating the emission function for free-free emission $\left(\epsilon_{\nu}^{f f}\right)$ over the volume of the stellar envelope. We improve upon this first-order calculation by also including the extinction $\left(e^{-\tau(\rho)}\right)$ factor in the integral over volume. In this way, we account for the reduction in optically thin emission with increasing wavelength. This gives us, replacing Equation 3,

$$
F_{\lambda}^{f f-\text { thin }}=7.12 \times 10^{-13}\left(\frac{n_{e} \mu \mathrm{m} \mathrm{pc}}{10^{11} \lambda D}\right)^{2}\left(\frac{10^{4}}{T_{s h}}\right)^{0.5}\left(\frac{R_{s h}}{10^{12}}\right)^{3}\left(e^{-1.44 \frac{1 \mu \mathrm{m}}{\lambda} \frac{10^{4}}{T_{s h}}}\right)\left(\frac{\int e^{-\tau(\rho)} d V}{V_{\text {full }}}\right)
$$

To fit our observations, we sum the emission from these three sources, varying the temperature $\left(T_{s h}\right)$, radius $\left(R_{s h}\right)$, and electron density $\left(n_{E}\right)$ of the shell. This sum is then compared to our observed spectrum. We calculate a reduced $\chi^{2}$ value based upon nine points in our observed spectra; these nine points located in regions at least $0.05 \mu \mathrm{m}$ from any of the observed emission lines, in order to not contaminate the $\chi^{2}$ with line emission. The flux at each point is determined by averaging the flux over $\lambda-0.05$ to $\lambda+0.05 \mu \mathrm{m}$, in order to reduce the effects of local noise spikes. The results of these fits are shown in Table 6. In Figure 4, we show the observed spectrum of $\gamma$ Cas with the overlaid fit. The observed spectrum is shown as a dotted line, the fit curve is shown as a solid line, the dashed line and dot-dash line are the optically thin and optically thick components of the emission respectively, the diamonds are the nine points used to calculate the reduced $\chi^{2}$, and the open triangle is the $12 \mu \mathrm{m}$ flux measured by IRAS. 


\subsection{Comparisons with Previous Work}

Two works in particular, Gehrz et al. 1974 and Waters et al. 1987, have calculated parameters of Be stellar envelopes. Gehrz et al. calculated $R_{s h}$ and $n_{e}$ from their observations, based upon an estimate of $T_{s h}$ (this estimate was constrained solely by the requirement that $T_{s h}$ be between $10000 \mathrm{~K}$ and $T_{\star}$ ) by using the estimated $T_{s h}$ and by calculating the wavelength where the extrapolations of optically thin and optically thick free-free emission intersect (at which point the optical depth $\left.\tau\left(\lambda_{c}\right) \equiv 1\right)$. This provides a relation between the radius of the disk and the electron density. Further, by assuming a Rayleigh-Jeans distribution for both the optically thick shell flux and the stellar flux (at $\lambda_{c}$ ), they obtain an expression for the radius of the shell, which then determines the electron density of the disk. For these calculations, they have assumed the disk to be flattened with an aspect ratio $A=r_{s} / d_{d i s k}=5$ (where $d_{d i s k}$ is the full thickness of the disk), the same value used for our modelling. As in our model, theirs includes the simplifying assumption that the electron density was uniform in the disk.

Waters et al. assume $T_{s h}=0.8 T_{\star}$ and calculate $R_{s h}$ and the density profile $(\rho(r))$ for the stellar disks using the "curve of growth" method (Lamers \& Waters 1984). For this method, they assume a disk with an opening angle $\theta$, a disk density which is proporational to $r^{-n}$, a density at the inner edge of the shell of $\rho_{o}$, and a disk radius $r_{s h}$ (the sharp outer cutoff to the disk). For their modelling, they have assumed a pole-on view of the disk, such that the opening angle $\theta$ is deprecated in their calculations; this is contrary to the both our assumption and the assumption of Gehrz et al. that the disk is viewed edge-on. They then use a combination of optically thin and optically thick free-free radiation as a comparision to the observed monochromatic infrared excess (as found from IRAS spectra and using assumed stellar parameters). By comparing the shapes of the observed excess and their models, they obtain a value fo the density parameter $n$, and from the shift required to match the observed and model curves they obtain $\rho_{o}$. These models are dependent upon the assumed shell radius, such that the authors run a series of models to find the best-fit combination of $n$ and $r_{s h}$, yielding the values used for comparision in this paper (notably, their values for $r_{s h}$ are all lower limits). We do not compare our values for $n_{e}$ with the $\rho(r)$ calculated by Waters et al. because of the large uncertainty in the mass densities they derived, the uncertainty in the proper ionization fraction of the envelope gas, and the dependence upon the exact shell radius.

We present the results of the modelling discussed in this paper, as well as the results of both Gehrz et al. and Waters et al. in Table 7 for ease of comparision. We find that our results are generally consistent (within factors of a few) with those of both authors. However, in a number of the cases, Waters et al. derive shell radii which are significantly larger than the radii derived here. This can be explained by the assumptions which have gone into our modelling, and the wavelength regimes of the observations. We have assumed an isothermal disk, whereas Waters et al. did not. Since their observations utilize IRAS data, they will be sensitive to the cool parts of the shell which should exist in the case of a thermal gradient. These discrepancies are particularly apparent in the cases of $\phi$ Per and $\gamma$ Cas, bright sources with accurate $12 \mu \mathrm{m}, 25 \mu \mathrm{m}$, and $60 \mu \mathrm{m}$ IRAS detections. 
Comparing our results to those of Gehrz et al., we find that in the case of $\zeta$ Tau we predict an electron density which is a factor of two below that of Gehrz et al., and a moderately larger disk. In the cases of $\gamma$ Cas and $\phi$ Per, we derive larger, hotter disks than Gehrz et al., but have similar electron densities. The simplicity of the models used by Gehrz et al. are most likely responsible for this discrepancy. They extrapolated curves for both the optically-thin and optically-thick segments of the free-free emission, then used the intercept point of these two curves to derive numerical values for radius and temperature of the shell. The improved modelling techniques used here should be better able to limit these same parameters.

In Table 7, we also show calculated total mass of hydrogen in the emitting disk. These numbers assume complete ionization of the hydrogen gas, such that $n_{e}=n_{H}$, and uniform density, an assumption already used in the modelling. We find that the masses of the disks are relatively small, ranging from $2.5 \times 10^{-11} \mathrm{M}_{\odot}(o$ Aqr $)$ to $8.1 \times 10^{-9} \mathrm{M}_{\odot}(\gamma \mathrm{Cas})$.

\section{Discussion}

These spectra present MIR features of Be stars and give some indications of the range of emission characteristics of them as well. Continuum emission is explained by a combination of optically-thick and optically-thin free-free emission. Both the continuum and line emission is likely to arise in a warm stellar envelope. The peculiar objects ( $\beta$ Lyr and MWC 349) display complicated line emission which is difficult to explain with simple models. In the case of $\beta$ Lyr, a relatively simple explanation for the presence of hydrogen and helium is possible, and the observed [Ne II] emission is easily explained as emission from a large gas cloud surrounding the binary system. In the case of MWC 349, the observed features are consistent with previous observations, and the observed forbidden emission implies a large volume of low-density ionized gas.

The authors would like to thank the staff at Palomar Observatory for their assistance. We would also like to thank S. Hony for sharing ISO data and results prior to publication. SAR acknowledges L.B.F.M. Waters and M. Simon for helpful discussions. This research was partly supported by NASA Contract 960803.

\section{REFERENCES}

Allen, D.A. \& Swings, J.P. 1976. A\&A, 47, 293.

Andrillat, Y., Jaschek, M. \& Jaschek, C. 1994. A\&AS, 103, 135.

Andrillat, Y. \& Swings, J.P. 1976. ApJ, 304, L123.

Brugel, E.W. \& Wallerstein, G. 1979. ApJ, 229, L23. 
Cohen, M., Bieging, J.H., Dreher, J.W., \& Welch, W.J. 1985. ApJ, 292, 249.

Cohen, M., Walker, R. G., Barlow, M.J., Deacon, J.R. 1992. AJ, 104, 1650.

Dachs, J. 1986. In "Physics of Be Stars", IAU Symposium 96, eds. A. Slettebak \& T. Snow, Reidel, Dordrecht.

De Greve, J.P. \& Linnell, A.P. 1994. A\&A, 291, 786.

Doazan \& Thomas 1982

Dougherty, S. \& Taylor, A. 1992. Nature, 359, 808.

Dougherty, S. \& Taylor, A. 1994. MNRAS, 269, 1123.

Gehrz, R., Hackwell, J. \& Jones, T. 1974. ApJ, 191, 675.

Gezari, D., Schmitz, M., Pitts, P., \& Mead, J. 1993. Catalog of Infrared Observations, Third Edition.

Haberl, F. 1995. A\&A, 296, 685.

Hamann, F. \& Simon, M. 1987. ApJ, 318, 356.

Hanuschik, R. 1996. A\&A, 308, 170.

Harmanec, P., Morand, F., Bonneau, D., Jiang, Y., Yang, S., Guinan, E.F., Hall, D.S., Mourard, D., Hadrava, P., Božić, H., Sterken, C., Tallon-Bosc, I., Walker, G.A.H., McCook, G.P., Vakili, F., Stee, Ph., \& Le Contel, J.M. 1996. A\&A, 312, 879.

Harmanec, P. \& Scholz, G. 1993. A\&A, 279, 131.

Hoffman, J.L., Nordsieck, K.H. \& Fox, G.K. 1998. AJ, 115, 1576.

Hony, S., Waters, L.B.F.M., Zaal, P.A., de Koter, A., Marlborough, J.M., Millar, C.E., Trams, N.R., Morris, P.W., \& de Graauw, Th. 1999. A\&A, submitted.

Hubeny, I., Harmanec, P., \& Shore, S.N. 1994. A\&A, 289, 411.

Hummer, D.G \& Storey, P.J. 1987. MNRAS, 224, 801.

Jared, M.M, Hilditch, R.W. \& Shillen, I. 1989. MNRAS, 238, 1085.

Johnson, H.L. 1978. Rev. Mex. Astron. \& Astrof. 4, 3.

Lamers, H.J.G.L.M. \& Waters, L.B.F.M. 1984. A\&A, 136, 37.

Lamers, H.J.G.L.M. \& Waters, L.B.F.M. 1987. A\&A, 182, 80.

Marlborough, J., Chen, H. \& Waters, L.B.F.M. 1993. ApJ, 408, 646. 
Marlborough, J.M., Zijlstra, J.-W., Waters, L.B.F.M. 1997. A\&A, 321, 867.

Pešek, I., Hefty, J. \& Skoupý, V. 1996. A\&AS, 118, 227.

Peters, G. 1982. In "Be stars", IAU Symp. 98, eds. M. Jaschek \& H.G. Groth, Reidel, Dordrecht, p. 353.

Poeckert, R. \& Marlborough, J. 1978. ApJ, 220, 940.

Poeckert, R. 1981. PASP, 93, 297.

Pols, O.R., Coté, J., Waters, L.B.F.M., \& Heise, J. 1991. A\&A, 241, 419.

Quirrenbach, A., Buscher, D., Mozurkewich, D., Hummel, C. \& Armstrong, J. 1994, A\&A, 283, L13.

Smith, J. D., Rinehart, S. A., Houck, J. R., Van Cleve, J. E., Wilson, J. C., Colonno, M., Schoenwald, J., Pirger, B., Blacken, C. 1998. Proc. SPIE, VOL, PAGE.

Stee, Ph., Vakili, F., Bonneau, D. \& Mourard, D. 1998. A\&A, 332, 268.

Slettebak, A. 1988. PASP, 100, 770.

Slettebak, A., Collins, G \& Traux, R. 1992. ApJS, 81, 335.

Smith, M.A. 1995. ApJ, 442, 812.

Strelnitski, V.S., Smith, H.A., Ponomarev, V.O. 1996. ApJ, 470, 1134.

Taylor, A.R., Dougherty, S.M., Waters, L.B.F.M., \& Bjorkman, K.S. A\&A, 231, 453.

Telting, J., Waters, L.B.F.M., Persi, P. \& Dunlop, S. 1993. A\&A, 270, 355.

Thompson, R.I., Strittmatter, P.A., Erickson, E.F, Witteborn, F.C., \& Strecker, D.W. 1977. ApJ, $218,170$.

Thum, C. \& Greve, A. 1997. A\&A, 324, 699.

Thum, C., Martin-Pintado, J., Quirrenbach, A., \& Matthews, H.E. 1998. A\&A, 333, L63.

Van Cleve, J., Gull, G. E., Rinehart, S. A., Smith, J. D., Wilson, J. C., Houck, J. R., Brown, R., Blalock, W. 1998. PASP, 110, 1479.

Waters, L.B.F.M. 1986. A\&A, 162, 121.

Waters, L.B.F.M. 1987. A\&A, 185, 206.

This preprint was prepared with the AAS LATEX macros v5.0. 
Table 1: Observations of Be Stars

\begin{tabular}{lllll}
\hline \hline Object & Date & $\begin{array}{l}\text { Integration } \\
\text { Time (s) }\end{array}$ & $\begin{array}{l}\text { Spectral } \\
\text { Type }\end{array}$ & $\begin{array}{l}\text { IRAS } \\
12 \mu \mathrm{m} \text { Flux (Jy) }\end{array}$ \\
\hline$\psi$ Per & Aug. 29, 1998 & 668 & B5Ve & \\
$\eta$ Tau & Aug. 29, 1998 & 653 & B7III & 4.32 \\
$\zeta$ Tau & Aug. 28, 1998 & 481 & B2IV & 8.30 \\
48 Per & Aug. 28, 1998 & 661 & B3Ve & 3.80 \\
$\phi$ Per & Aug. 27, 1998 & 741 & B2Vpe & \\
$\gamma$ Cas & Aug. 27, 1998 & 240 & B0IVe & 18.83 \\
$o$ Aqr & Aug. 27, 1998 & 586 & B7IVe & 1.18 \\
$\beta$ Lyr & July 5, 1998 & 930 & B7Ve+ & 5.01 \\
& July 13, 1998 & & & \\
EW Lac & July 12, 1998 & 661 & B3IVpe & \\
$\kappa$ Dra & July 5, 1998 & 1094 & B6IIIpe & 4.12 \\
MWC 349 & July 12, 1998 & 481 & Bpe & 179.0 \\
& Aug. 27, 1998 & & & \\
\hline
\end{tabular}


Table 2A. Data on observed lines $-\psi$ Per

\begin{tabular}{lcccc}
\hline \hline Transition & $\begin{array}{c}\lambda_{\text {theory }} \\
(\mu \mathrm{m})\end{array}$ & $\begin{array}{c}\lambda_{\text {obs }} \\
(\mu \mathrm{m})\end{array}$ & $\begin{array}{c}\Delta \lambda \\
\left(10^{-3} \mu \mathrm{m}\right)\end{array}$ & $\begin{array}{c}F_{\nu} \\
\left(10^{-19} \mathrm{~W} \mathrm{~cm}^{-2} \mu \mathrm{m}^{-1}\right)\end{array}$ \\
\hline H 14 $\rightarrow 9$ & 12.587 & $12.582 \pm 0.0064$ & $13.40 \pm 5.833$ & $6.73 \pm 3.96$ \\
H 19 $\rightarrow 10$ & 12.611 & $12.613 \pm 0.0024$ & $4.06 \pm 2.622$ & $3.04 \pm 2.93$ \\
H 11 $\rightarrow 8$ & 12.387 & $12.391 \pm 0.0047$ & $3.55 \pm 4.034$ & $2.81 \pm 4.21$ \\
H 7 $\rightarrow 6$ & 12.372 & $12.371 \pm 0.0016$ & $7.18 \pm 1.236$ & $10.60 \pm 2.43$ \\
H 21 $\rightarrow 10$ & 11.792 & $11.787 \pm 0.0046$ & $8.93 \pm 4.136$ & $4.14 \pm 2.56$ \\
H 15 $\rightarrow 9$ & 11.540 & $11.540 \pm 0.0042$ & $13.10 \pm 4.098$ & $7.83 \pm 3.09$ \\
H 9 $\rightarrow 7$ & 11.309 & $11.308 \pm 0.0013$ & $82.50 \pm 1.216$ & $9.86 \pm 1.91$ \\
H 24 $\rightarrow 10$ & 11.033 & $11.033 \pm 0.0049$ & $6.91 \pm 4.302$ & $2.82 \pm 2.24$ \\
H 25 $\rightarrow 10$ & 10.855 & $10.854 \pm 0.0041$ & $5.78 \pm 3.545$ & $2.55 \pm 1.89$ \\
H 16 $\rightarrow 9$ & 10.804 & $10.803 \pm 0.0018$ & $7.52 \pm 1.640$ & $7.09 \pm 2.03$ \\
H 26 $\rightarrow 10$ & 10.701 & $10.695 \pm 0.0053$ & $8.00 \pm 1.414$ & $3.03 \pm 1.67$ \\
H 12 $\rightarrow 8$ & 10.504 & $10.502 \pm 0.0021$ & $8.11 \pm 1.920$ & $5.97 \pm 1.90$ \\
H 17 $\rightarrow 9$ & 10.261 & $10.256 \pm 0.0032$ & $12.90 \pm 2.921$ & $7.87 \pm 2.40$ \\
H 30 $\rightarrow 10$ & 10.258 & & Blended with $17 \rightarrow 9$ & \\
H 18 $\rightarrow 9$ & 9.847 & $9.842 \pm 0.0035$ & $8.42 \pm 3.154$ & $6.40 \pm 3.09$ \\
H 19 $\rightarrow 9$ & 9.522 & $9.518 \pm 0.0043$ & $6.66 \pm 3.782$ & $3.31 \pm 2.59$ \\
H 13 $\rightarrow 8$ & 9.392 & $9.390 \pm 0.0013$ & $6.11 \pm 1.192$ & $9.35 \pm 2.29$ \\
H 20 $\rightarrow 9$ & 9.261 & $9.258 \pm 0.0051$ & $8.63 \pm 4.691$ & $4.39 \pm 3.10$ \\
H 21 $\rightarrow 9$ & 9.047 & $9.044 \pm 0.0046$ & $4.35 \pm 3.810$ & $2.36 \pm 2.57$ \\
H 10 $\rightarrow 7$ & 8.760 & $8.758 \pm 0.0012$ & $4.97 \pm 1.373$ & $7.77 \pm 2.78$ \\
H 14 $\rightarrow 8$ & 8.665 & $8.663 \pm 0.0022$ & $8.41 \pm 1.997$ & $10.34 \pm 3.23$ \\
H 24 $\rightarrow 9$ & 8.594 & $8.594 \pm 0.0033$ & $4.61 \pm 3.466$ & $3.75 \pm 3.47$ \\
\hline
\end{tabular}


Table 2B. Data on observed lines $-\eta$ Tau

\begin{tabular}{|c|c|c|c|c|}
\hline Transition & $\begin{array}{c}\lambda_{\text {theory }} \\
(\mu \mathrm{m})\end{array}$ & $\begin{array}{l}\lambda_{o b s} \\
(\mu \mathrm{m})\end{array}$ & $\begin{array}{c}\Delta \lambda \\
\left(10^{-3} \mu \mathrm{m}\right)\end{array}$ & $\begin{array}{c}F_{\nu} \\
\left(10^{-19} \mathrm{~W} \mathrm{~cm}^{-2} \mu \mathrm{m}^{-1}\right)\end{array}$ \\
\hline Н $14 \rightarrow 9$ & 12.611 & $12.586 \pm 0.0032$ & $9.14 \pm 3.075$ & $5.07 \pm 2.2 .8$ \\
\hline H $11 \rightarrow 8$ & 12.387 & $12.393 \pm 0.0016$ & $4.21 \pm 1.782$ & $2.80 \pm 1.74$ \\
\hline $\mathrm{H} 7 \rightarrow 6$ & 12.372 & $12.375 \pm 0.0010$ & $5.68 \pm 1.392$ & $6.76 \pm 2.07$ \\
\hline H $15 \rightarrow 9$ & 11.540 & $11.538 \pm 0.0026$ & $8.84 \pm 2.376$ & $5.27 \pm 1.86$ \\
\hline $\mathrm{H} 9 \rightarrow 7$ & 11.309 & $11.308 \pm 0.0009$ & $7.41 \pm 0.870$ & $8.67 \pm 1.33$ \\
\hline H $23 \rightarrow 10$ & 11.243 & $11.245 \pm 0.0070$ & $\ldots$ & \\
\hline H $24 \rightarrow 10$ & 11.033 & $11.044 \pm 0.0093$ & $\ldots$ & .. \\
\hline H $25 \rightarrow 10$ & 10.855 & $10.859 \pm 0.0042$ & $6.02 \pm 5.527$ & $1.75 \pm 2.17$ \\
\hline H $16 \rightarrow 9$ & 10.804 & $10.802 \pm 0.0018$ & $7.74 \pm 1.621$ & $6.50 \pm 1.79$ \\
\hline H $12 \rightarrow 8$ & 10.504 & $10.501 \pm 0.0016$ & $7.65 \pm 1.483$ & $5.61 \pm 1.43$ \\
\hline Н $17 \rightarrow 9$ & 10.261 & $10.257 \pm 0.0028$ & $10.00 \pm 2.586$ & $4.97 \pm 1.73$ \\
\hline H $18 \rightarrow 9$ & 9.847 & $9.844 \pm 0.0026$ & $6.72 \pm 2.346$ & $4.43 \pm 2.05$ \\
\hline H $19 \rightarrow 9$ & 9.522 & $9.516 \pm 0.0026$ & $5.23 \pm 2.019$ & $3.10 \pm 1.65$ \\
\hline H $13 \rightarrow 8$ & 9.392 & $9.389 \pm 0.0019$ & $3.14 \pm 2.605$ & $2.62 \pm 2.89$ \\
\hline $\mathrm{H} 10 \rightarrow 7$ & 8.760 & $8.758 \pm 0.0016$ & $6.42 \pm 1.429$ & $9.55 \pm 2.80$ \\
\hline H $14 \rightarrow 8$ & 8.665 & $8.663 \pm 0.0023$ & $7.19 \pm 2.113$ & $8.19 \pm 3.22$ \\
\hline Н $24 \rightarrow 9$ & 8.594 & $8.595 \pm 0.0084$ & $\ldots$ & \\
\hline H $15 \rightarrow 8$ & 8.155 & $8.164 \pm 0.0017$ & $5.57 \pm 1.506$ & $7.33 \pm 2.65$ \\
\hline Н $29 \rightarrow 9$ & 8.173 & & Blended with & $15 \rightarrow 8$ \\
\hline
\end{tabular}


Table 2C. Data on observed lines $-\zeta$ Tau

\begin{tabular}{|c|c|c|c|c|}
\hline Transition & $\begin{array}{c}\lambda_{\text {theory }} \\
(\mu \mathrm{m})\end{array}$ & $\begin{array}{l}\lambda_{o b s} \\
(\mu \mathrm{m})\end{array}$ & $\begin{array}{c}\Delta \lambda \\
\left(10^{-3} \mu \mathrm{m}\right)\end{array}$ & $\begin{array}{c}F_{\nu} \\
\left(10^{-19} \mathrm{~W} \mathrm{~cm}^{-2} \mu \mathrm{m}^{-1}\right)\end{array}$ \\
\hline H $18 \rightarrow 10$ & 13.188 & $13.191 \pm 0.0025$ & $7.09 \pm 2.144$ & $4.41 \pm 1.76$ \\
\hline H $19 \rightarrow 10$ & 12.611 & $12.611 \pm 0.0036$ & $8.54 \pm 3.164$ & $5.16 \pm 2.27$ \\
\hline Н $14 \rightarrow 9$ & 12.587 & $12.586 \pm 0.0032$ & $6.88 \pm 3.412$ & $4.23 \pm 2.42$ \\
\hline H $11 \rightarrow 8$ & 12.387 & $12.395 \pm 0.0062$ & $3.80 \pm 9.182$ & $0.91 \pm 2.40$ \\
\hline $\mathrm{H} 7 \rightarrow 6$ & 12.372 & $12.375 \pm 0.0039$ & $11.60 \pm 3.078$ & $8.93 \pm 2.89$ \\
\hline H $20 \rightarrow 10$ & 12.157 & $12.160 \pm 0.0027$ & $7.94 \pm 2.588$ & $4.52 \pm 1.94$ \\
\hline H $21 \rightarrow 10$ & 11.792 & $11.796 \pm 0.0030$ & $10.40 \pm 2.794$ & $5.05 \pm 1.76$ \\
\hline H $15 \rightarrow 9$ & 11.540 & $11.542 \pm 0.0025$ & $10.00 \pm 2.219$ & $6.20 \pm 1.83$ \\
\hline H $22 \rightarrow 10$ & 11.492 & $11.493 \pm 0.0040$ & $8.41 \pm 3.562$ & $2.93 \pm 1.67$ \\
\hline $\mathrm{H} 9 \rightarrow 7$ & 11.309 & $11.310 \pm 0.0022$ & $8.51 \pm 2.032$ & $5.46 \pm 1.73$ \\
\hline H $23 \rightarrow 10$ & 11.243 & $11.243 \pm 0.0032$ & $7.54 \pm 2.775$ & $3.53 \pm 1.72$ \\
\hline H $24 \rightarrow 10$ & 11.033 & $11.037 \pm 0.0037$ & $6.13 \pm 4.111$ & $2.56 \pm 2.31$ \\
\hline H $25 \rightarrow 10$ & 10.855 & $10.860 \pm 0.0037$ & $7.93 \pm 2.941$ & $3.95 \pm 1.93$ \\
\hline H $16 \rightarrow 9$ & 10.804 & $10.804 \pm 0.0016$ & $9.71 \pm 1.481$ & $10.54 \pm 2.12$ \\
\hline H $26 \rightarrow 10$ & 10.701 & $10.696 \pm 0.0036$ & $4.66 \pm 4.525$ & $2.08 \pm 2.53$ \\
\hline H $27 \rightarrow 10$ & 10.567 & $10.573 \pm 0.0058$ & $4.18 \pm 5.796$ & $0.88 \pm 1.57$ \\
\hline $\mathrm{H} 12 \rightarrow 8$ & 10.504 & $10.507 \pm 0.0039$ & $6.73 \pm 3.551$ & $3.74 \pm 2.41$ \\
\hline H $28 \rightarrow 10$ & 10.451 & $10.458 \pm 0.0080$ & $6.84 \pm 7.271$ & $1.91 \pm 2.48$ \\
\hline H $29 \rightarrow 10$ & 10.348 & $10.353 \pm 0.0113$ & & \\
\hline Н $17 \rightarrow 9$ & 10.261 & $10.264 \pm 0.0016$ & $7.96 \pm 1.505$ & $8.71 \pm 2.18$ \\
\hline H $30 \rightarrow 10$ & 10.258 & & Blended with & $17 \rightarrow 9$ \\
\hline H $31 \rightarrow 10$ & 10.177 & $10.178 \pm 0.0024$ & $3.84 \pm 2.404$ & $1.89 \pm 1.65$ \\
\hline H $18 \rightarrow 9$ & 9.847 & $9.849 \pm 0.0016$ & $7.23 \pm 1.451$ & $9.15 \pm 2.39$ \\
\hline H $19 \rightarrow 9$ & 9.522 & $9.527 \pm 0.0010$ & & \\
\hline H $13 \rightarrow 8$ & 9.392 & $9.395 \pm 0.0019$ & $7.21 \pm 1.708$ & $9.64 \pm 3.01$ \\
\hline H $20 \rightarrow 9$ & 9.261 & $9.266 \pm 0.0018$ & $5.88 \pm 1.719$ & $7.34 \pm 2.84$ \\
\hline H $21 \rightarrow 9$ & 9.047 & $9.049 \pm 0.0036$ & $6.44 \pm 3.288$ & $5.61 \pm 3.65$ \\
\hline $\mathrm{H} 22 \rightarrow 9$ & 8.870 & $8.875 \pm 0.0035$ & $5.78 \pm 2.963$ & $4.66 \pm 3.22$ \\
\hline $\mathrm{H} 10 \rightarrow 7$ & 8.760 & $8.761 \pm 0.0024$ & $5.72 \pm 1.965$ & $5.45 \pm 3.03$ \\
\hline $\mathrm{H} 23 \rightarrow 9$ & 8.721 & $8.723 \pm 0.0025$ & $6.64 \pm 2.232$ & $7.86 \pm 3.46$ \\
\hline $\mathrm{H} 14 \rightarrow 8$ & 8.665 & $8.667 \pm 0.0024$ & $6.96 \pm 2.207$ & $8.30 \pm 3.48$ \\
\hline $\mathrm{H} 24 \rightarrow 9$ & 8.594 & $8.596 \pm 0.0031$ & $5.84 \pm 2.774$ & $6.67 \pm 4.02$ \\
\hline $\mathrm{H} 25 \rightarrow 9$ & 8.485 & $8.488 \pm 0.0021$ & $2.75 \pm 2.296$ & $3.02 \pm 3.51$ \\
\hline $\mathrm{H} 26 \rightarrow 9$ & 8.391 & $8.392 \pm 0.0033$ & $4.99 \pm 2.427$ & $4.60 \pm 3.26$ \\
\hline $\mathrm{H} 27 \rightarrow 9$ & 8.309 & $8.306 \pm 0.0036$ & $4.37 \pm 3.477$ & $3.55 \pm 3.58$ \\
\hline $\mathrm{H} 28 \rightarrow 9$ & 8.236 & $8.239 \pm 0.0011$ & $\ldots$ & $\ldots$ \\
\hline $\mathrm{H} 15 \rightarrow 8$ & 8.155 & $8.156 \pm 0.0009$ & $4.85 \pm 1.035$ & $11.02 \pm 3.38$ \\
\hline H $29 \rightarrow 9$ & 8.173 & \multicolumn{3}{|c|}{ Blended with $15 \rightarrow 8$} \\
\hline $\mathrm{H} 30 \rightarrow 9$ & 8.155 & $8.103 \pm 0.0023$ & $6.33 \pm 2.102$ & $8.12 \pm 3.74$ \\
\hline
\end{tabular}


Table 2D. Data on observed lines - 48 Per

\begin{tabular}{|c|c|c|c|c|}
\hline Transition & $\begin{array}{c}\lambda_{\text {theory }} \\
(\mu \mathrm{m})\end{array}$ & $\begin{array}{l}\lambda_{o b s} \\
(\mu \mathrm{m})\end{array}$ & $\begin{array}{c}\Delta \lambda \\
\left(10^{-3} \mu \mathrm{m}\right)\end{array}$ & $\begin{array}{c}F_{\nu} \\
\left(10^{-19} \mathrm{~W} \mathrm{~cm}^{-2} \mu \mathrm{m}^{-1}\right)\end{array}$ \\
\hline H $18 \rightarrow 10$ & 13.188 & $13.188 \pm 0.0065$ & $6.14 \pm 4.530$ & $3.04 \pm 2.95$ \\
\hline $\mathrm{H} 19 \rightarrow 10$ & 12.611 & \multicolumn{3}{|c|}{ Blended with $\mathrm{H} 14 \rightarrow 9$} \\
\hline $\mathrm{H} 14 \rightarrow 9$ & 12.587 & $12.587 \pm 0.0028$ & $9.00 \pm 2.685$ & $6.27 \pm 2.42$ \\
\hline $\mathrm{H} 11 \rightarrow 8$ & 12.387 & \multicolumn{3}{|c|}{ Blended with $\mathrm{H} 7 \rightarrow 6$} \\
\hline $\mathrm{H} 7 \rightarrow 6$ & 12.372 & $12.379 \pm 0.0019$ & $8.33 \pm 1.594$ & $7.72 \pm 2.01$ \\
\hline H $20 \rightarrow 10$ & 12.157 & $12.159 \pm 0.0033$ & $5.90 \pm 3.344$ & $2.30 \pm 1.66$ \\
\hline $\mathrm{H} 21 \rightarrow 10$ & 11.792 & $11.792 \pm 0.0061$ & $10.10 \pm 5.602$ & $2.66 \pm 1.92$ \\
\hline $\mathrm{H} 15 \rightarrow 9$ & 11.540 & $11.537 \pm 0.0027$ & $8.52 \pm 2.482$ & $4.18 \pm 1.60$ \\
\hline $\mathrm{H} 9 \rightarrow 7$ & 11.309 & $11.306 \pm 0.0011$ & $6.41 \pm 0.889$ & $6.00 \pm 1.12$ \\
\hline $\mathrm{H} 23 \rightarrow 10$ & 11.243 & $11.236 \pm 0.0062$ & $7.72 \pm 5.487$ & $1.69 \pm 1.60$ \\
\hline $\mathrm{H} 24 \rightarrow 10$ & 11.033 & $11.028 \pm 0.0044$ & $4.16 \pm 8.134$ & $0.95 \pm 2.39$ \\
\hline $\mathrm{H} 16 \rightarrow 9$ & 10.804 & $10.802 \pm 0.0019$ & $7.31 \pm 1.777$ & $4.82 \pm 1.56$ \\
\hline H $27 \rightarrow 10$ & 10.567 & $10.566 \pm 0.0197$ & $\ldots$ & \\
\hline H $12 \rightarrow 8$ & 10.504 & $10.501 \pm 0.0015$ & $6.79 \pm 1.334$ & $5.05 \pm 1.35$ \\
\hline $\mathrm{H} 28 \rightarrow 10$ & 10.451 & $10.446 \pm 0.0226$ & $\ldots$ & $\ldots$ \\
\hline Н $17 \rightarrow 9$ & 10.261 & $10.257 \pm 0.0013$ & $7.61 \pm 1.179$ & $6.69 \pm 1.37$ \\
\hline $\mathrm{H} 18 \rightarrow 9$ & 9.847 & $9.846 \pm 0.0021$ & $6.19 \pm 1.812$ & $4.92 \pm 1.94$ \\
\hline $\mathrm{H} 19 \rightarrow 9$ & 9.522 & $9.517 \pm 0.0019$ & $4.36 \pm 1.390$ & $3.65 \pm 1.54$ \\
\hline $\mathrm{H} 13 \rightarrow 8$ & 9.392 & $9.388 \pm 0.0011$ & $5.92 \pm 0.913$ & $8.08 \pm 1.64$ \\
\hline $\mathrm{H} 20 \rightarrow 9$ & 9.261 & $9.260 \pm 0.0049$ & $9.45 \pm 4.608$ & $4.07 \pm 2.60$ \\
\hline H $21 \rightarrow 9$ & 9.047 & $9.044 \pm 0.0046$ & $6.15 \pm 4.097$ & $3.14 \pm 2.68$ \\
\hline $\mathrm{H} 22 \rightarrow 9$ & 8.870 & $8.870 \pm 0.0051$ & $2.58 \pm 5.069$ & $1.33 \pm 2.77$ \\
\hline $\mathrm{H} 10 \rightarrow 7$ & 8.760 & $8.757 \pm 0.0009$ & $5.82 \pm 0.756$ & $9.98 \pm 1.70$ \\
\hline $\mathrm{H} 23 \rightarrow 9$ & 8.721 & $8.718 \pm 0.0059$ & $5.76 \pm 5.464$ & $2.14 \pm 2.60$ \\
\hline $\mathrm{H} 14 \rightarrow 8$ & 8.665 & $8.662 \pm 0.0015$ & $5.68 \pm 1.286$ & $7.55 \pm 2.27$ \\
\hline
\end{tabular}


Table 2E. Data on observed lines $-\phi$ Per

\begin{tabular}{|c|c|c|c|c|}
\hline Transition & $\begin{array}{c}\lambda_{\text {theory }} \\
(\mu \mathrm{m})\end{array}$ & $\begin{array}{c}\lambda_{o b s} \\
(\mu \mathrm{m})\end{array}$ & $\begin{array}{c}\Delta \lambda \\
\left(10^{-3} \mu \mathrm{m}\right)\end{array}$ & $\begin{array}{c}F_{\nu} \\
\left(10^{-19} \mathrm{~W} \mathrm{~cm}^{-2} \mu \mathrm{m}^{-1}\right)\end{array}$ \\
\hline $\mathrm{H} 18 \rightarrow 10$ & 13.188 & $13.185 \pm 0.0059$ & $13.10 \pm 5.435$ & $4.23 \pm 2.29$ \\
\hline $\mathrm{H} 19 \rightarrow 10$ & 12.611 & $12.615 \pm 0.0009$ & $3.84 \pm 1.099$ & $2.53 \pm 1.21$ \\
\hline Н $14 \rightarrow 9$ & 12.587 & $12.588 \pm 0.0024$ & $6.90 \pm 1.996$ & $2.91 \pm 1.11$ \\
\hline $\mathrm{H} 11 \rightarrow 8$ & 12.387 & \multicolumn{3}{|c|}{ Blended with $7 \rightarrow 6$} \\
\hline $\mathrm{H} 7 \rightarrow 6$ & 12.372 & $12.364 \pm 0.0040$ & $12.49 \pm 3.691$ & $4.41 \pm 1.71$ \\
\hline $\mathrm{H} 20 \rightarrow 10$ & 12.157 & $12.155 \pm 0.0048$ & $12.20 \pm 4.486$ & $2.88 \pm 1.38$ \\
\hline H $15 \rightarrow 9$ & 11.540 & $11.536 \pm 0.0020$ & $7.88 \pm 1.774$ & $3.29 \pm 0.97$ \\
\hline $\mathrm{H} 9 \rightarrow 7$ & 11.309 & $11.305 \pm 0.0024$ & $69.90 \pm 2.115$ & $2.38 \pm 0.95$ \\
\hline $\mathrm{H} 23 \rightarrow 10$ & 11.243 & $11.238 \pm 0.0039$ & $6.00 \pm 3.846$ & $1.33 \pm 1.04$ \\
\hline H $24 \rightarrow 10$ & 11.033 & $11.033 \pm 0.0052$ & $9.45 \pm 4.707$ & $2.20 \pm 1.47$ \\
\hline H $16 \rightarrow 9$ & 10.804 & $10.801 \pm 0.0019$ & $7.68 \pm 1.812$ & $4.24 \pm 1.35$ \\
\hline H $26 \rightarrow 10$ & 10.701 & $10.698 \pm 0.0036$ & $5.10 \pm 2.802$ & $1.63 \pm 1.17$ \\
\hline H $27 \rightarrow 10$ & 10.567 & $10.577 \pm 0.0058$ & $6.70 \pm 4.402$ & $1.17 \pm 1.05$ \\
\hline $\mathrm{H} 12 \rightarrow 8$ & 10.504 & $10.502 \pm 0.0023$ & $6.04 \pm 2.163$ & $2.55 \pm 1.27$ \\
\hline H $28 \rightarrow 10$ & 10.451 & $10.452 \pm 0.0067$ & $4.690 \pm 6.250$ & $7.24 \pm 1.22$ \\
\hline $\mathrm{H} 17 \rightarrow 9$ & 10.261 & $10.258 \pm 0.0018$ & $7.51 \pm 1.642$ & $4.47 \pm 1.29$ \\
\hline H $30 \rightarrow 10$ & 10.258 & \multicolumn{3}{|c|}{ Blended with $17 \rightarrow 9$} \\
\hline $\mathrm{H} 18 \rightarrow 9$ & 9.847 & $9.845 \pm 0.0017$ & $5.29 \pm 1.330$ & $5.25 \pm 1.56$ \\
\hline $\mathrm{H} 19 \rightarrow 9$ & 9.522 & $9.518 \pm 0.0038$ & $5.59 \pm 3.424$ & $2.92 \pm 2.07$ \\
\hline $\mathrm{H} 13 \rightarrow 8$ & 9.392 & $9.391 \pm 0.0017$ & $5.27 \pm 1.597$ & $4.55 \pm 1.79$ \\
\hline H $20 \rightarrow 9$ & 9.261 & $9.257 \pm 0.0026$ & $5.67 \pm 2.591$ & $3.49 \pm 2.02$ \\
\hline Н $21 \rightarrow 9$ & 9.047 & $9.047 \pm 0.0022$ & $5.20 \pm 2.006$ & $3.49 \pm 1.76$ \\
\hline H $22 \rightarrow 9$ & 8.870 & $8.871 \pm 0.0073$ & $10.41 \pm 7.766$ & $6.53 \pm 5.99$ \\
\hline $\mathrm{H} 10 \rightarrow 7$ & 8.760 & $8.758 \pm 0.0043$ & $5.43 \pm 5.002$ & $3.40 \pm 4.26$ \\
\hline Н $29 \rightarrow 9$ & 8.173 & \multicolumn{3}{|c|}{ Blended with $15 \rightarrow 8$} \\
\hline H $15 \rightarrow 8$ & 8.155 & $8.152 \pm 0.0014$ & $3.81 \pm 1.021$ & $7.0299 \pm 2.5343$ \\
\hline
\end{tabular}


Table 2. Data on observed lines

\begin{tabular}{|c|c|c|c|c|}
\hline Transition & $\lambda_{\text {theory }}$ & $\lambda_{o b s}$ & as, $\operatorname{Aug}_{\Delta \lambda} 1998$ & $F_{\nu}$ \\
\hline H 7 & 12.372 & $12.369 \pm 0.0031$ & $9.53 \pm 2.643$ & $1.09 \pm 0.363$ \\
\hline & & $.307 \pm 0.0015$ & $6.57 \pm$ & $1.20 \pm$ \\
\hline $\mathrm{H} 10 \rightarrow 7$ & 8.760 & $.759 \pm 0.0031$ & $6.13 \pm 2.804$ & $2.19 \pm 1.328$ \\
\hline $\mathrm{H} 11 \rightarrow 8$ & 12.387 & $2.394 \pm 0.0065$ & $7.71 \pm 6.266$ & $0.45 \pm 0.433$ \\
\hline H 1 & & $.504 \pm 0.0018$ & $6.85 \pm$ & $1.25 \pm$ \\
\hline & & $3 \pm 0$ & $6.85 \pm$ & $1.79 \pm$ \\
\hline H $14 \rightarrow 8$ & & & $7.69 \pm 2.543$ & $2.33 \pm 1.174$ \\
\hline $\mathrm{H} 14 \rightarrow 9$ & 2.587 & $2.589 \pm 0.0022$ & $6.37 \pm 1.716$ & $1.05 \pm 0.370$ \\
\hline & & $8.154 \pm 0.0010$ & $4.20 \pm 0.832$ & $4.96 \pm$ \\
\hline & & 17 & $8.03 \pm$ & 1.11 \\
\hline & & & $6.39 \pm$ & 83 \\
\hline & 51 & 18 & $7.62 \pm$ & $1.28 \pm$ \\
\hline & 847 & $846 \pm 0.0016$ & $6.38 \pm 1$ & $1.74 \pm($ \\
\hline & & & $6.70 \pm$ & $0.74 \pm$ \\
\hline & & & $2.70=$ & \\
\hline & 1 & & $5.63 \pm$ & 615 \\
\hline H 20 & 9.261 & \pm 0.00 & $9.17 \pm$ & $0.88 \pm$ \\
\hline$\rightarrow 10$ & 12.1 & 44 & $9.53 \pm$ & $0.46 \pm$ \\
\hline & & & $2.27=$ & \\
\hline & & & $7.66=$ & 34 \\
\hline & & & $6.64 \pm$ & $1.34 \pm$ \\
\hline H $22 \rightarrow 10$ & 11.492 & $11.488 \pm 0.0032$ & $4.14 \pm 4.405$ & $0.36 \pm 0.537$ \\
\hline & 8.721 & $8.726 \pm 0.0172$ & & \\
\hline & & & $7.70=$ & $0.39 \exists$ \\
\hline & & & 2.10 & 2.02 \\
\hline H $24 \rightarrow 10$ & & $4 \pm 0.0061$ & $8.01 \pm$ & $0.43 \pm 0.383$ \\
\hline H $25 \rightarrow 9$ & 105 & \pm 0.0050 & $6.74 \pm 4.387$ & $1.43 \pm 1.237$ \\
\hline & & & & \\
\hline & & 6 & $4.28 \pm 3.611$ & $1.61 \pm 1.737$ \\
\hline & & & & \\
\hline Н $27 \rightarrow 9$ & & 3 & & $\cdots$ \\
\hline $\mathrm{H} 27 \rightarrow 10$ & & $.580 \pm 0.0091$ & & \\
\hline & & $8.240 \pm 0.0018$ & $3.65 \pm 1.244$ & $3.58 \pm 1.550$ \\
\hline & & & & \\
\hline H $29 \rightarrow 9$ & 8.173 & \multicolumn{3}{|c|}{ Blended with $15 \rightarrow 8$} \\
\hline Н $29 \rightarrow 10$ & 10.348 & $0.334 \pm 0.0049$ & & \\
\hline $\mathrm{H} 30 \rightarrow 9$ & 8.116 & $8.107 \pm 0.0040$ & $6.80 \pm 3.654$ & $1.71 \pm 1.218$ \\
\hline & & \multicolumn{3}{|c|}{ Blended with $17 \rightarrow 9$} \\
\hline $\mathrm{H} 31 \rightarrow 10$ & 10.177 & $10.181 \pm 0.0074$ & $6.71 \pm 6.115$ & $0.28 \pm 0.332$ \\
\hline
\end{tabular}


Table 2G. Data on observed lines - o Aqr

\begin{tabular}{lcccc}
\hline \hline Transition & $\begin{array}{c}\lambda_{\text {theory }} \\
(\mu \mathrm{m})\end{array}$ & $\begin{array}{c}\lambda_{\text {obs }} \\
(\mu \mathrm{m})\end{array}$ & $\begin{array}{c}\Delta \lambda \\
\left(10^{-3} \mu \mathrm{m}\right)\end{array}$ & $\begin{array}{c}F_{\nu} \\
\left(10^{-19} \mathrm{~W} \mathrm{~cm}^{-2} \mu \mathrm{m}^{-1}\right)\end{array}$ \\
\hline H 19 $\rightarrow 10$ & 12.611 & $12.618 \pm 0.0162$ & $9.24 \pm 13.21$ & $2.03 \pm 19.08$ \\
H 14 $\rightarrow 9$ & 12.587 & $12.590 \pm 0.0041$ & $10.84 \pm 3.713$ & $2.78 \pm 1.23$ \\
H 11 $\rightarrow 8$ & 12.387 & $12.384 \pm 0.0084$ & $21.00 \pm 7.290$ & $4.24 \pm 1.98$ \\
H 7 $\rightarrow 6$ & 12.372 & $12.370 \pm 0.0027$ & $5.44 \pm 1.812$ & $1.86 \pm 0.83$ \\
H 21 $\rightarrow 10$ & 11.792 & $11.782 \pm 0.0061$ & $12.13 \pm 5.769$ & $1.43 \pm 0.94$ \\
H 15 $\rightarrow 9$ & 11.540 & $11.543 \pm 0.0035$ & $9.75 \pm 3.229$ & $18.11 \pm 6.02$ \\
H 9 $\rightarrow 7$ & 11.309 & $11.307 \pm 0.0020$ & $9.35 \pm 1.813$ & $2.68 \pm 0.69$ \\
H 24 $\rightarrow 10$ & 11.033 & $11.024 \pm 0.0081$ & $\ldots$ & $\cdots$ \\
H 16 $\rightarrow 9$ & 10.804 & $10.805 \pm 0.0036$ & $7.73 \pm 3.211$ & $1.33 \pm 0.72$ \\
H 12 $\rightarrow 8$ & 10.504 & $10.502 \pm 0.0013$ & $7.26 \pm 1.142$ & $2.89 \pm 0.60$ \\
H 28 $\rightarrow 10$ & 10.451 & $10.453 \pm 0.0264$ & $\cdots$ & $\cdots$ \\
H 29 $\rightarrow 10$ & 10.348 & $10.343 \pm 0.0020$ & $\ldots$ & $\cdots$ \\
H 17 $\rightarrow 9$ & 10.261 & $10.260 \pm 0.0015$ & $4.80 \pm 1.005$ & $1.72 \pm 0.48$ \\
H 30 $\rightarrow 10$ & 10.258 & & Blended with $17 \rightarrow 9$ & \\
H 18 $\rightarrow 9$ & 9.847 & $9.846 \pm 0.0029$ & $4.70 \pm 2.315$ & $1.33 \pm 0.81$ \\
H 13 $\rightarrow 8$ & 9.392 & $9.392 \pm 0.0023$ & $8.80 \pm 2.075$ & $3.30 \pm 1.04$ \\
H 22 $\rightarrow 9$ & 8.870 & $8.871 \pm 0.0116$ & $\ldots$ & $\cdots$ \\
H 10 $\rightarrow 7$ & 8.760 & $8.759 \pm 0.0026$ & $7.86 \pm 2.421$ & $4.61 \pm 1.86$ \\
H 14 $\rightarrow 8$ & 8.665 & $8.668 \pm 0.0024$ & $5.10 \pm 2.145$ & $3.00 \pm 1.67$ \\
H 15 $\rightarrow 8$ & 8.155 & $8.155 \pm 0.0011$ & $4.43 \pm 1.011$ & $4.68 \pm 1.40$ \\
H 29 $\rightarrow 9$ & 8.173 & & Blended with $15 \rightarrow 8$ & \\
\hline
\end{tabular}


Table 2H. Data on observed lines - EW Lac

\begin{tabular}{|c|c|c|c|c|}
\hline Transition & $\begin{array}{c}\lambda_{\text {theory }} \\
(\mu \mathrm{m})\end{array}$ & $\begin{array}{l}\lambda_{o b s} \\
(\mu \mathrm{m})\end{array}$ & $\begin{array}{c}\Delta \lambda \\
\left(10^{-3} \mu \mathrm{m}\right)\end{array}$ & $\begin{array}{c}F_{\nu} \\
\left.\mathrm{W} \mathrm{cm}-2 \mu \mathrm{m}^{-1}\right)\end{array}$ \\
\hline H $19 \rightarrow 10$ & 12.611 & $12.614 \pm 0.0076$ & $8.70 \pm 6.928$ & $1.24 \pm 1.16$ \\
\hline $\mathrm{H} 14 \rightarrow 9$ & 12.587 & $12.591 \pm 0.0045$ & $8.40 \pm 3.521$ & $2.03 \pm 1.05$ \\
\hline H $11 \rightarrow 8$ & 12.387 & & Blended with $\mathrm{H} 7 \rightarrow 6$ & \\
\hline $\mathrm{H} 7 \rightarrow 6$ & 12.372 & $12.373 \pm 0.0017$ & $12.40 \pm 1.549$ & $3.73 \pm 0.62$ \\
\hline $\mathrm{H} 15 \rightarrow 9$ & 11.540 & $11.543 \pm 0.0026$ & $8.20 \pm 2.307$ & $0.88 \pm 0.70$ \\
\hline $\mathrm{H} 22 \rightarrow 10$ & 11.492 & $11.491 \pm 0.0100$ & & \\
\hline $\mathrm{H} 9 \rightarrow 7$ & 11.309 & $11.308 \pm 0.0017$ & $8.40 \pm 1.575$ & $1.66 \pm 0.41$ \\
\hline Н $16 \rightarrow 9$ & 10.804 & $10.805 \pm 0.0023$ & $6.60 \pm 1.876$ & $0.68 \pm 0.27$ \\
\hline $\mathrm{H} 26 \rightarrow 10$ & 10.701 & $10.696 \pm 0.0029$ & $4.83 \pm 3.181$ & $0.39 \pm 0.32$ \\
\hline H $12 \rightarrow 8$ & 10.504 & $10.500 \pm 0.0021$ & $7.60 \pm 1.871$ & $1.09 \pm 0.36$ \\
\hline Н $17 \rightarrow 9$ & 10.261 & $10.263 \pm 0.0027$ & $14.00 \pm 2.669$ & $1.78 \pm 1.16$ \\
\hline H $30 \rightarrow 10$ & 10.258 & & Blended with $17 \rightarrow 9$ & \\
\hline Н $19 \rightarrow 9$ & 9.522 & $9.523 \pm 0.0024$ & $5.50 \pm 2.010$ & $1.41 \pm 1.44$ \\
\hline $\mathrm{H} 13 \rightarrow 8$ & 9.392 & $9.386 \pm 0.0026$ & $5.70 \pm 2.528$ & $1.11 \pm 1.39$ \\
\hline H $20 \rightarrow 9$ & 9.261 & $9.254 \pm 0.0043$ & $3.86 \pm 2.720$ & $5.50 \pm 4.00$ \\
\hline Н $21 \rightarrow 9$ & 9.047 & $9.048 \pm 0.0027$ & $2.50 \pm 3.141$ & $3.96 \pm 5.02$ \\
\hline H $10 \rightarrow 7$ & 8.760 & $8.758 \pm 0.0014$ & $6.10 \pm 1.321$ & $1.79 \pm 1.11$ \\
\hline H $14 \rightarrow 8$ & 8.665 & $8.664 \pm 0.0031$ & $6.60 \pm 2.555$ & $0.85 \pm 0.97$ \\
\hline
\end{tabular}


Table 2I. Data on observed lines $-\kappa$ Dra

\begin{tabular}{lcccc}
\hline \hline Transition & $\begin{array}{c}\lambda_{\text {theory }} \\
(\mu \mathrm{m})\end{array}$ & $\begin{array}{c}\lambda_{\text {obs }} \\
(\mu \mathrm{m})\end{array}$ & $\begin{array}{c}\Delta \lambda \\
\left(10^{-3} \mu \mathrm{m}\right)\end{array}$ & $\begin{array}{c}F_{\nu} \\
\left(10^{-19} \mathrm{~W} \mathrm{~cm}^{-2} \mu \mathrm{m}^{-1}\right)\end{array}$ \\
\hline H 18 $\rightarrow 10$ & 13.188 & $13.192 \pm 0.0069$ & $6.30 \pm 7.434$ & $1.67 \pm 2.47$ \\
H 19 $\rightarrow 10$ & 12.611 & $12.617 \pm 0.0026$ & $7.50 \pm 2.327$ & $1.34 \pm 1.73$ \\
H 14 $\rightarrow 9$ & 12.587 & $12.590 \pm 0.0041$ & $8.40 \pm 3.497$ & $2.56 \pm 2.30$ \\
H 11 $\rightarrow 8$ & 12.387 & $12.393 \pm 0.0026$ & $3.54 \pm 3.874$ & $0.18 \pm 2.83$ \\
H 7 $\rightarrow 6$ & 12.372 & $12.376 \pm 0.0017$ & $7.55 \pm 1.727$ & $0.35 \pm 1.75$ \\
H 20 $\rightarrow 10$ & 12.157 & $12.155 \pm 0.0046$ & $6.93 \pm 3.672$ & $1.07 \pm 1.73$ \\
H 21 $\rightarrow 10$ & 11.792 & $11.793 \pm 0.0059$ & $12.70 \pm 5.418$ & $1.96 \pm 2.42$ \\
H 15 $\rightarrow 9$ & 11.540 & $11.539 \pm 0.0021$ & $10.30 \pm 1.897$ & $3.03 \pm 1.61$ \\
H 22 $\rightarrow 10$ & 11.492 & $11.491 \pm 0.0055$ & $8.00 \pm 4.943$ & $0.90 \pm 0.75$ \\
H 9 $\rightarrow 7$ & 11.309 & $11.309 \pm 0.0014$ & $7.60 \pm 1.341$ & $2.75 \pm 1.35$ \\
H 23 $\rightarrow 10$ & 11.243 & $11.244 \pm 0.0061$ & $7.80 \pm 5.390$ & $0.87 \pm 1.76$ \\
H 25 $\rightarrow 10$ & 10.855 & $10.847 \pm 0.0042$ & $8.01 \pm 12.14$ & $1.16 \pm 2.33$ \\
H 16 $\rightarrow 9$ & 10.804 & $10.803 \pm 0.0014$ & $79.40 \pm 1.301$ & $3.39 \pm 1.61$ \\
H 26 $\rightarrow 10$ & 10.701 & $10.701 \pm 0.0027$ & $\ldots$ & $\cdots$ \\
H 12 $\rightarrow 8$ & 10.504 & $10.504 \pm 0.0017$ & $8.00 \pm 1.520$ & $3.10 \pm 1.70$ \\
H 17 $\rightarrow 9$ & 10.261 & $10.263 \pm 0.0017$ & $8.50 \pm 1.528$ & $3.33 \pm 1.74$ \\
H 18 $\rightarrow 9$ & 9.847 & $9.845 \pm 0.0022$ & $6.92 \pm 1.979$ & $2.71 \pm 1.03$ \\
H 19 $\rightarrow 9$ & 9.522 & $9.521 \pm 0.0023$ & $8.57 \pm 2.067$ & $3.49 \pm 1.11$ \\
H 13 $\rightarrow 8$ & 9.392 & $9.390 \pm 0.0014$ & $6.75 \pm 1.202$ & $3.84 \pm 0.93$ \\
H 20 $\rightarrow 9$ & 9.261 & $9.260 \pm 0.0034$ & $5.20 \pm 2.654$ & $12.16 \pm 6.23$ \\
H 21 $\rightarrow 9$ & 9.047 & $9.047 \pm 0.0033$ & $7.82 \pm 2.956$ & $2.59 \pm 1.27$ \\
H 10 $\rightarrow 7$ & 8.760 & $8.760 \pm 0.0012$ & $5.75 \pm 1.054$ & $3.81 \pm 0.93$ \\
H 14 $\rightarrow 8$ & 8.665 & $8.666 \pm 0.0018$ & $7.48 \pm 1.604$ & $3.92 \pm 1.12$ \\
H 24 $\rightarrow 9$ & 8.594 & $8.592 \pm 0.0026$ & $\ldots$ & \\
H 15 $\rightarrow 8$ & 8.155 & $8.158 \pm 0.0009$ & $6.49 \pm 0.814$ & $6.58 \pm 1.09$ \\
H 29 $\rightarrow 9$ & 8.173 & & Blended with $15 \rightarrow 8$ & \\
\hline
\end{tabular}


Table 3A. Data on observed lines $-\beta$ Lyr

\begin{tabular}{|c|c|c|c|c|}
\hline Transition $^{\mathrm{a}}$ & $\begin{array}{c}\lambda_{\text {theory }} \\
(\mu \mathrm{m})\end{array}$ & $\begin{array}{l}\lambda_{o b s} \\
(\mu \mathrm{m})\end{array}$ & $\begin{array}{c}\Delta \lambda \\
\left(10^{-3} \mu \mathrm{m}\right)\end{array}$ & $\begin{array}{c}F_{\nu} \\
\left(10^{-19} \mathrm{~W} \mathrm{~cm}^{-2} \mu \mathrm{m}^{-1}\right)\end{array}$ \\
\hline$[\mathrm{Ne} \mathrm{II}]$ & 12.81 & $12.813 \pm 0.0017$ & $9.91 \pm 1.565$ & $8.83 \pm 1.84$ \\
\hline H $14 \rightarrow 9$ & 12.587 & $12.583 \pm 0.0039$ & $10.60 \pm 3.615$ & $5.08 \pm 2.26$ \\
\hline H $11 \rightarrow 8$ & 12.387 & $12.393 \pm 0.0035$ & $6.84 \pm 3.384$ & $4.09 \pm 2.31$ \\
\hline $\mathrm{H} 7 \rightarrow 6$ & 12.372 & $12.368 \pm 0.0020$ & $10.16 \pm 1.851$ & $38.90 \pm 8.70$ \\
\hline $\mathrm{H} 9 \rightarrow 7$ & 11.309 & $11.305 \pm 0.0004$ & $9.86 \pm 0.389$ & $20.81 \pm 1.09$ \\
\hline He I 4P-4S, 3Po-3S & 10.882 & $10.882 \pm 0.0010$ & $8.69 \pm 0.953$ & $14.24 \pm 2.07$ \\
\hline H $16 \rightarrow 9$ & 10.804 & $10.801 \pm 0.0071$ & $6.85 \pm 6.682$ & $2.18 \pm 2.65$ \\
\hline $\mathrm{H} 12 \rightarrow 8$ & 10.504 & $10.504 \pm 0.0014$ & $10.55 \pm 1.306$ & $14.23 \pm 2.34$ \\
\hline Н $17 \rightarrow 9$ & 10.261 & $10.263 \pm 0.0066$ & $7.37 \pm 5.992$ & $2.45 \pm 2.58$ \\
\hline $\mathrm{H} 18 \rightarrow 9$ & 9.847 & $9.846 \pm 0.0028$ & $8.62 \pm 2.576$ & $3.12 \pm 1.25$ \\
\hline$?$ & & $9.597 \pm 0.0012$ & $4.34 \pm 1.562$ & $2.49 \pm 1.12$ \\
\hline Н $19 \rightarrow 9$ & 9.522 & $9.516 \pm 0.0027$ & $5.74 \pm 2.232$ & $2.70 \pm 1.44$ \\
\hline $\mathrm{H} 13 \rightarrow 8$ & 9.392 & $9.389 \pm 0.0012$ & $9.77 \pm 1.029$ & $10.31 \pm 1.43$ \\
\hline $\mathrm{H} 10 \rightarrow 7$ & 8.760 & $8.758 \pm 0.0007$ & $8.14 \pm 0.608$ & $23.92 \pm 2.36$ \\
\hline H $14 \rightarrow 8$ & 8.665 & $8.663 \pm 0.0030$ & $7.64 \pm 2.760$ & $7.68 \pm 3.53$ \\
\hline H $15 \rightarrow 8$ & 8.155 & $8.153 \pm 0.0020$ & $5.11 \pm 1.780$ & $6.37 \pm 2.75$ \\
\hline
\end{tabular}

aThe hydrogen recombination lines may also be blended with the identical He I recombination transitions. 
Table 3B. Data on observed lines - MWC 349

\begin{tabular}{lcccc}
\hline \hline Transition & $\begin{array}{c}\lambda_{\text {theory }} \\
(\mu \mathrm{m})\end{array}$ & $\begin{array}{c}\lambda_{\text {obs }} \\
(\mu \mathrm{m})\end{array}$ & $\begin{array}{c}\Delta \lambda \\
\left(10^{-3} \mu \mathrm{m}\right)\end{array}$ & $\begin{array}{c}F_{\nu} \\
\left(10^{-17} \mathrm{~W} \mathrm{~cm}^{-2} \mu \mathrm{m}^{-1}\right)\end{array}$ \\
\hline$[\mathrm{Ne} \mathrm{II}]$ & 12.81 & $12.813 \pm 0.0002$ & $9.55 \pm 0.168$ & $95.35 \pm 2.21$ \\
$\mathrm{H} 7 \rightarrow 6$ & 12.372 & $12.372 \pm 0.0017$ & $13.35 \pm 1.534$ & $10.80 \pm 1.64$ \\
$?$ & $\ldots$ & $11.918 \pm 0.0045$ & $14.39 \pm 4.390$ & $5.89 \pm 2.32$ \\
$\mathrm{H} 9 \rightarrow 7$ & 11.309 & $11.310 \pm 0.0008$ & $7.83 \pm 0.771$ & $0.82 \pm 0.70$ \\
$\mathrm{H} 12 \rightarrow 8$ & 10.504 & $10.508 \pm 0.0025$ & $9.98 \pm 2.329$ & $0.06 \pm 1.18$ \\
\hline
\end{tabular}


Table 4: Properties of our Sources

\begin{tabular}{|c|c|c|c|c|c|}
\hline Object & $\begin{array}{l}\text { Shell } \\
\text { Star }\end{array}$ & $\begin{array}{c}\text { Spectroscopic } \\
\text { Binary }\end{array}$ & $\begin{array}{l}\text { X-Ray } \\
\text { Binary }\end{array}$ & $\begin{array}{l}\text { Radio } \\
\text { Source }\end{array}$ & References \\
\hline$\psi \mathrm{Per}$ & yes & no & no & yes & 1,7 \\
\hline$\eta$ Tau & yes & yes & no & yes & $1,3,9$ \\
\hline$\zeta \mathrm{Tau}$ & yes & yes & no & no & 1,3 \\
\hline 48 Per & no & yes & yes & no & 2,6 \\
\hline$\phi$ Per & yes & yes & no & no & 1 \\
\hline$\gamma$ Cas & yes & no & yes & yes & $1,4,9$ \\
\hline$o$ Aqr & yes & no & no & no & 1 \\
\hline EW Lac & yes & no & no & yes & 1,9 \\
\hline$\kappa$ Dra & no & yes & yes & no & 5,6 \\
\hline$\beta$ Lyr & no & yes & no & yes & 5,10 \\
\hline MWC 349 & no & no & no & yes & 8 \\
\hline
\end{tabular}

(1) Slettebak et al. 1992, (2) Poeckert 1981, (3) Jared et al. 1989, (4) Haberl 1995, (5) Pols et al. 1991, (6) Peters 1982, (7) Marlborough et al. 1997, (8) Strelnitski et al. 1996, (9) Taylor et al. 1990, (10)Pešek et al. 1996. 
Table 5. Stellar Parameters for Sources

\begin{tabular}{lclllll}
\hline \hline Object & $\begin{array}{c}T_{\text {eff }} \\
(\mathrm{K})\end{array}$ & $\begin{array}{c}R_{\star} \\
\left(\mathrm{R}_{\odot}\right)\end{array}$ & $\begin{array}{c}D \\
(\mathrm{pc})\end{array}$ & $\begin{array}{c}\log L \\
\left(\log L_{\odot}\right)\end{array}$ & $\begin{array}{c}1.25 \mu \mathrm{m} \\
(\mathrm{mag})\end{array}$ & $\begin{array}{l}2.2 \mu \mathrm{m} \\
(\mathrm{mag})\end{array}$ \\
\hline$\psi$ Per & 16000 & 7.0 & 215 & 3.20 & 4.30 & 3.94 \\
$\eta$ Tau & 14000 & 6.5 & 113 & 2.86 & 2.95 & 2.96 \\
$\zeta$ Tau & 16500 & 6.5 & 128 & 3.05 & 3.20 & 2.94 \\
48 Per & 16000 & 6.3 & 170 & 3.60 & $3.99^{\mathrm{a}}$ & $3.79^{\mathrm{b}}$ \\
$\phi$ Per & 20000 & 7.5 & 220 & 3.20 & 3.83 & 3.25 \\
$\gamma$ Cas & 31000 & 10.0 & 188 & 4.93 & 2.47 & 2.20 \\
$o$ Aqr & 11000 & 4.0 & 117 & 2.45 & 4.84 & 4.80 \\
EW Lac & 16000 & 6.5 & 337 & 3.54 & 5.34 & 5.00 \\
$\kappa$ Dra & 14500 & 6.4 & 153 & 2.70 & 3.86 & 3.75 \\
\hline
\end{tabular}

${ }^{\mathrm{a}}$ At $1.1 \mu \mathrm{m}$.

${ }^{\mathrm{b}}$ At $2.3 \mu \mathrm{m}$. 
Table 6. Parameter Values for Free-Free Fits

\begin{tabular}{lcccc}
\hline \hline Object & $\begin{array}{c}T_{s h} \\
\left(10^{4} \mathrm{~K}\right)\end{array}$ & $\begin{array}{c}n_{e} \\
\left(10^{11} \mathrm{~cm}^{-3}\right)\end{array}$ & $\begin{array}{c}R_{s h} \\
\left(\mathrm{R}_{\star}\right)\end{array}$ & $\chi^{2}$ \\
\hline$\psi$ Per & $1.60(+.07 /-.15)$ & $2.60(+.16 /-.07)$ & $4.61(+.21 /-.20)$ & 1.56 \\
$\eta$ Tau & $0.80(+.02 /-.06)$ & $1.52(+.08 /-.03)$ & $3.07( \pm .15)$ & 1.04 \\
$\zeta$ Tau & $1.39(+.04 /-.10)$ & $2.30(+.11 /-.04)$ & $4.95( \pm .32)$ & 4.27 \\
48 Per & $1.29(+.05 /-.08)$ & $2.21(+.09 /-.05)$ & $4.04( \pm .13)$ & 1.05 \\
$\phi$ Per & $1.65(+.06 /-.08)$ & $3.01(+.19 /-.10)$ & $5.02(+.10 /-.11)$ & 0.74 \\
$\gamma$ Cas & $2.68(+.15 /-.14)$ & $4.57(+.33 /-.21)$ & $6.00( \pm .13)$ & 1.12 \\
$o$ Aqr & $1.01(+.02 /-.11)$ & $1.60(+.05 /-.03)$ & $2.99(+.11 /-.13)$ & 0.54 \\
EW Lac & $1.32(+.02 /-.09)$ & $2.20(+.08 /-.03)$ & $3.80(+.20 /-.22)$ & 1.49 \\
$\kappa$ Dra & $1.20(+.04 /-.11)$ & $1.83(+.14 /-.04)$ & $3.12(+.14 /-.16)$ & 2.13 \\
\hline
\end{tabular}


Table 7. Comparision of Free-Free Fits

\begin{tabular}{llllllll}
\hline \hline Object & Author & $\begin{array}{c}T_{\star} \\
\left(10^{4} \mathrm{~K}\right)\end{array}$ & $\begin{array}{c}R_{\star} \\
\left(\mathrm{R}_{\odot}\right)\end{array}$ & $\begin{array}{c}T_{s h} \\
\left(10^{4} \mathrm{~K}\right)\end{array}$ & $\begin{array}{c}R_{s h} \\
\left(\mathrm{R}_{\odot}\right)\end{array}$ & $\begin{array}{c}n_{e} \\
\left(10^{11} \mathrm{~cm}^{-3}\right)\end{array}$ & $\begin{array}{c}\mathrm{M}_{H} \\
\left(10^{-10} \mathrm{M}_{\odot}\right)\end{array}$ \\
\hline$\psi$ Per & RHS & 1.6 & 7.0 & 1.6 & 32.3 & 2.60 & 9.3 \\
& WCL & 1.5 & 6.0 & 1.2 & 37.2 & $\ldots$ & \\
$\eta$ Tau & RHS & 1.4 & 6.5 & 0.8 & 20.0 & 1.52 & 0.93 \\
$\zeta$ Tau & RHS & 1.65 & 6.5 & 1.39 & 32.2 & 2.30 & 8.3 \\
& GHJ & 1.8 & 5.75 & 1.4 & 20.1 & 4.4 & \\
& WCL & 1.4 & 6.0 & 1.1 & 28.8 & $\ldots$ & 3.7 \\
48 Per & RHS & 1.6 & 6.3 & 1.29 & 25.5 & 2.21 & \\
& WCL & 1.8 & 6.5 & 1.45 & 36.4 & $\ldots$ & \\
$\phi$ Per & RHS & 2.0 & 7.5 & 1.65 & 37.7 & 3.01 & \\
& GHJ & 1.6 & 5.0 & 1.2 & 23.0 & 3.8 & \\
& WCL & 2.0 & 7.0 & 1.6 & 91.0 & $\ldots$ & \\
$\gamma$ Cas & RHS & 3.1 & 9.0 & 2.68 & 54.0 & 4.57 & \\
& GHJ & 2.6 & 7.2 & 2.0 & 29.5 & 4.4 & \\
& WCL & 3.1 & 10.0 & 2.5 & 80.0 & $\ldots$ & \\
\multirow{5}{*}{ Aqr } & RHS & 1.1 & 4.0 & 1.01 & 12.0 & 1.9 & \\
& WCL & 1.3 & 3.4 & 1.05 & 9.5 & $\ldots$ & \\
EW Lac & RHS & 1.6 & 6.5 & 1.32 & 24.7 & 2.20 & 3.2 \\
& WCL & 1.4 & 5.7 & 1.1 & 27.4 & $\ldots$ & \\
$\kappa$ Dra & RHS & 1.45 & 6.5 & 1.20 & 20.3 & 1.83 & 1.2 \\
& WCL & 1.3 & 4.5 & 1.05 & 22.1 & $\cdots$ & \\
& & & & & & & \\
\hline
\end{tabular}

${ }^{\mathrm{a}} \mathrm{WCL}=$ Waters et al. $1987, \mathrm{GHJ}=$ Gehrz et al 1974, and RHS $=$ this work. 

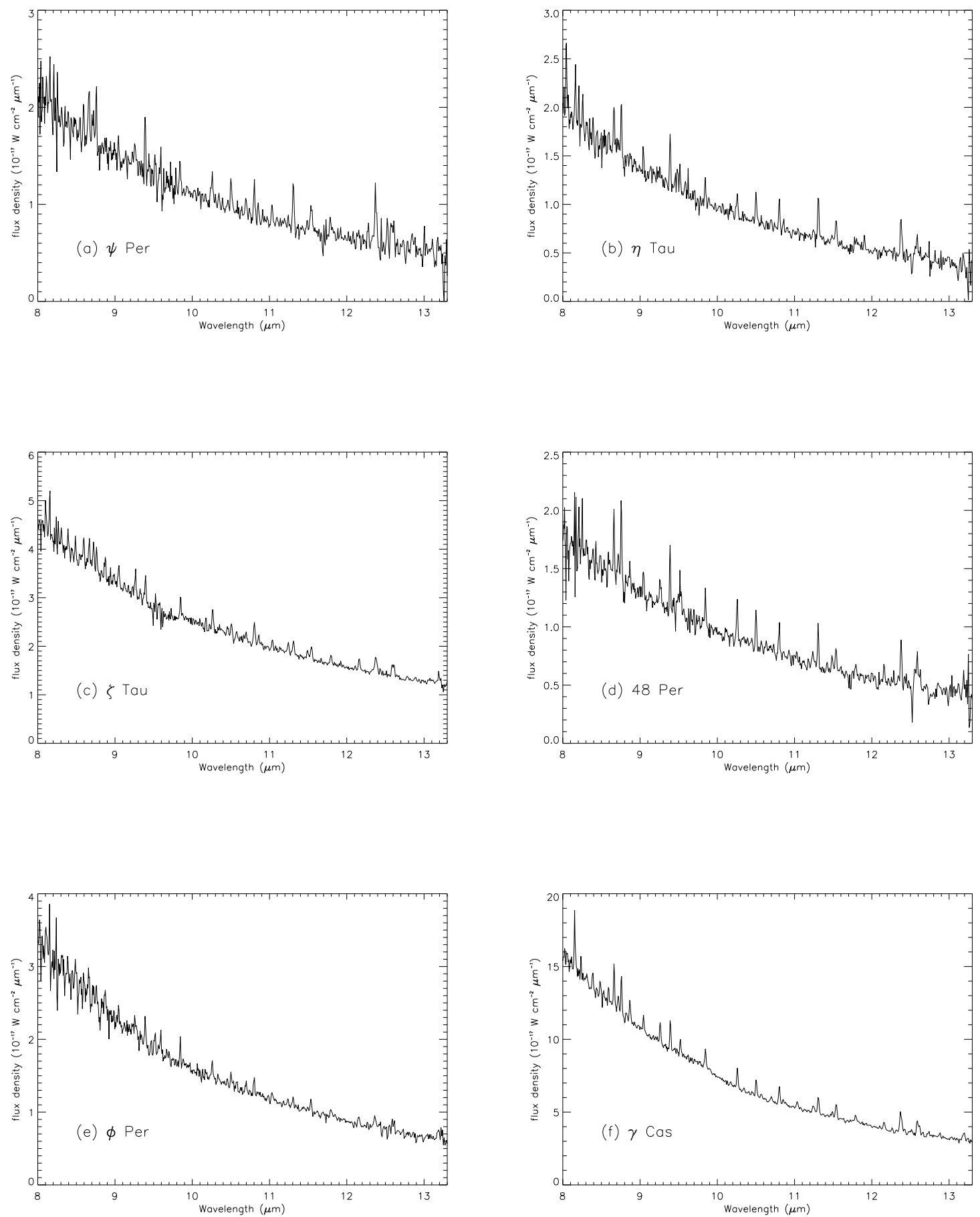

Fig. 1. - The reduced spectra of our sources, (a) $\Psi$ Per, (b) $\eta$ Tau, (c) $\zeta$ Tau, (d) 48 Per, (e) $\phi$ Per, (f) $\gamma$ Cas. 

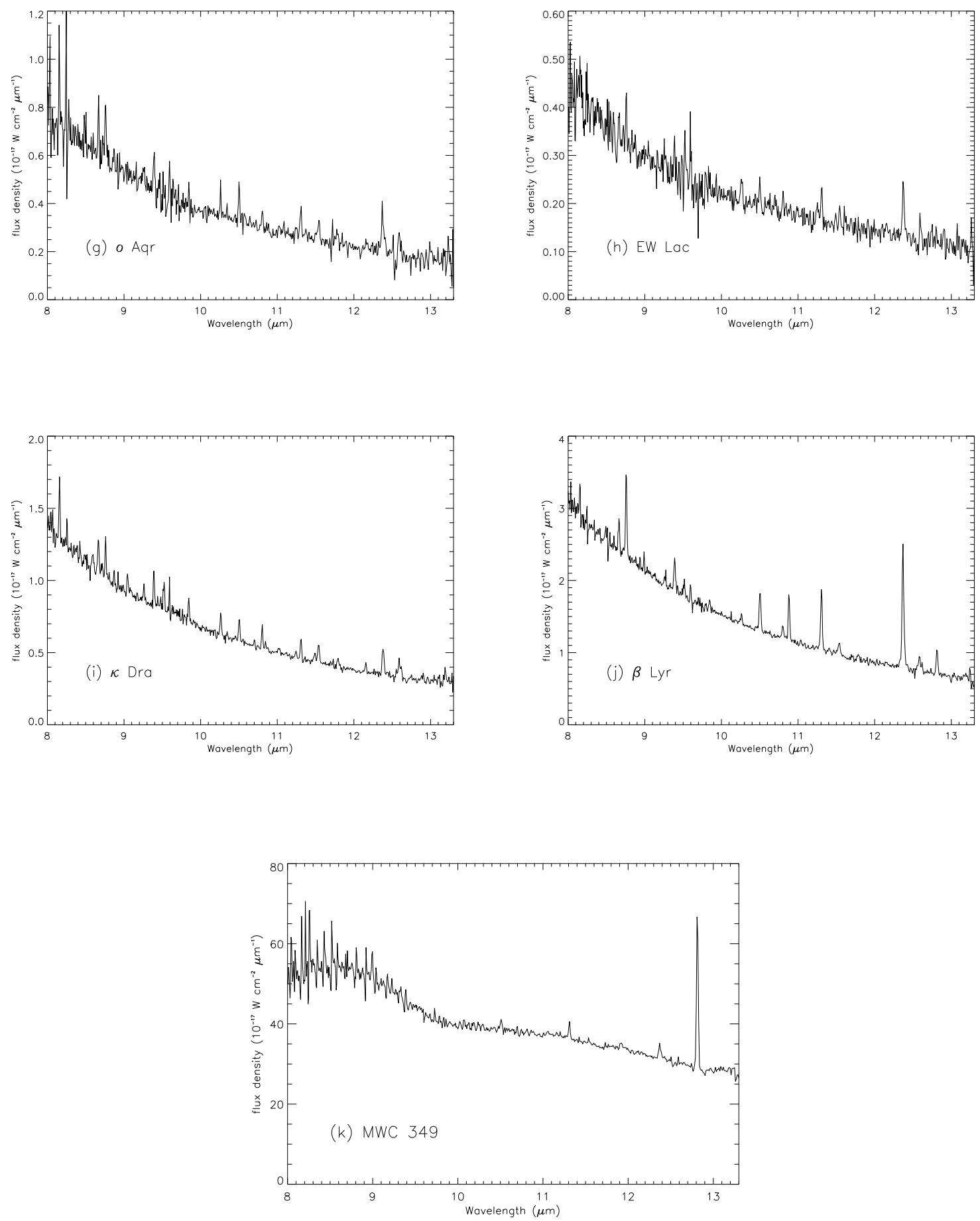

Fig. 1, Continued.- The reduced spectra of our sources, continued. (g) o Aqr, (h) EW Lac, (i) $\kappa$ Dra, (j) $\beta$ Lyr, and (k) MWC 349. 


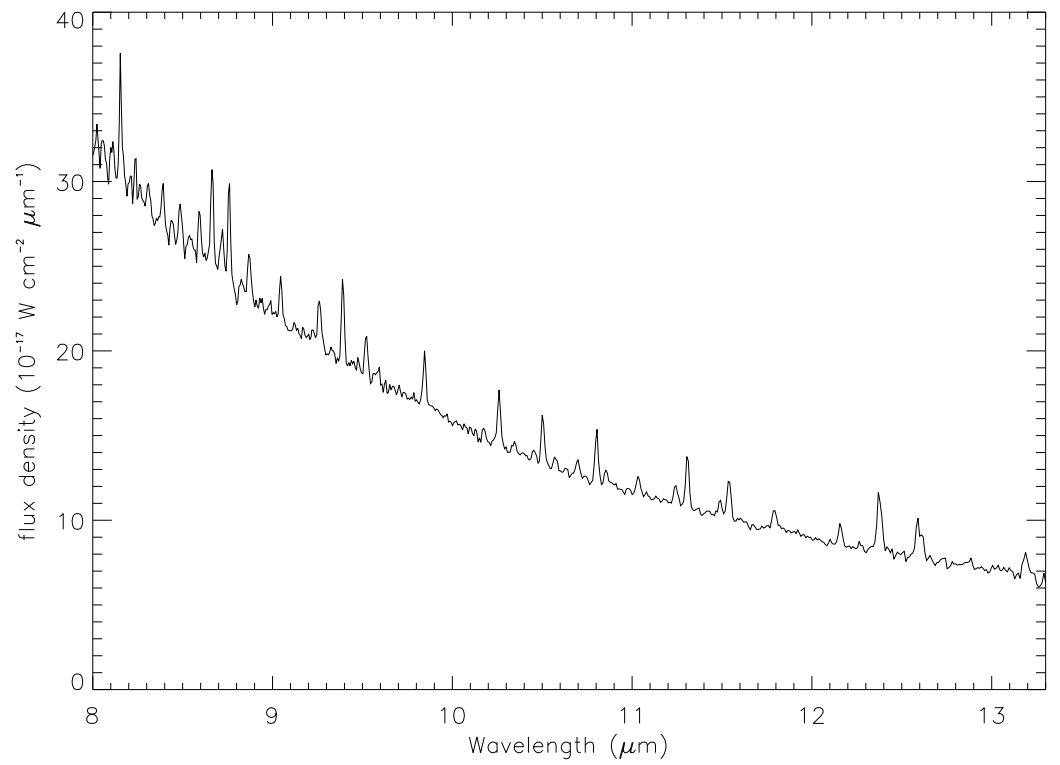

Fig. 2.- The spectrum produced by coadding all nine of the hydrogen spectra (HS) Be star spectra. Several lines which are visible in the individual spectra become clearer in this summed spectra.

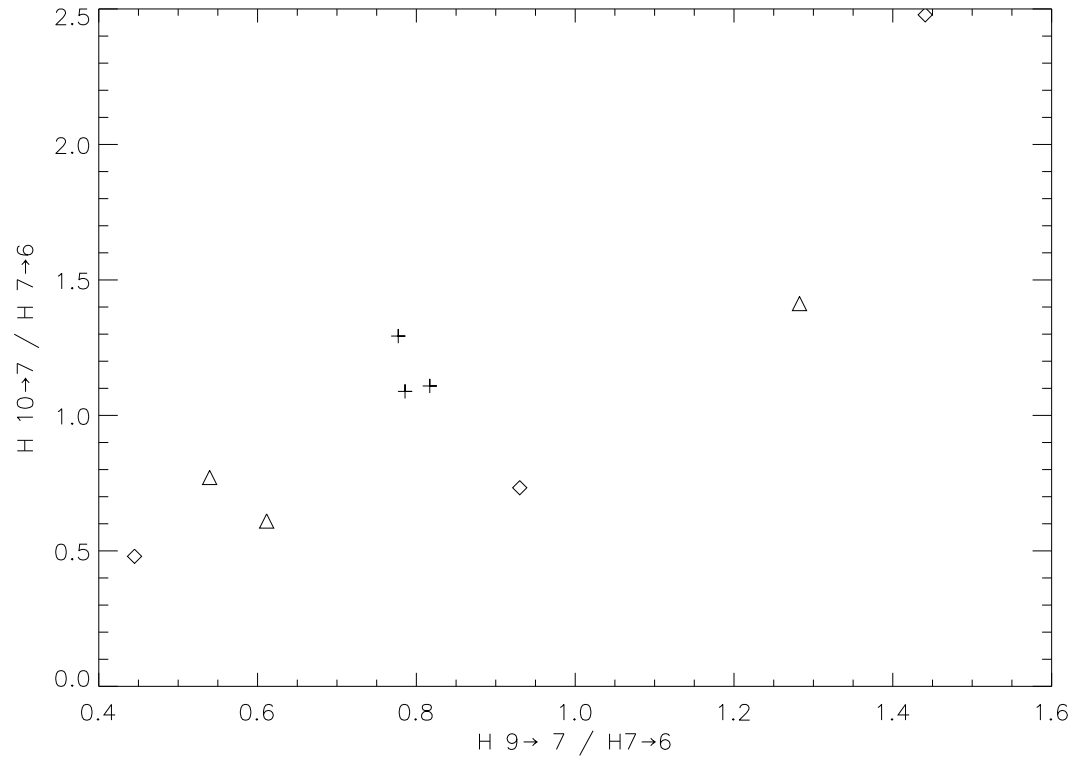

Fig. 3.- A comparison plot of the $\mathrm{H} 9 \rightarrow 7 / \mathrm{H} 7 \rightarrow 6$ ratio versus the $\mathrm{H} 10 \rightarrow 7 / \mathrm{H} 7 \rightarrow 6$ ratio. No strong correlation can be seen in this small sample set. 


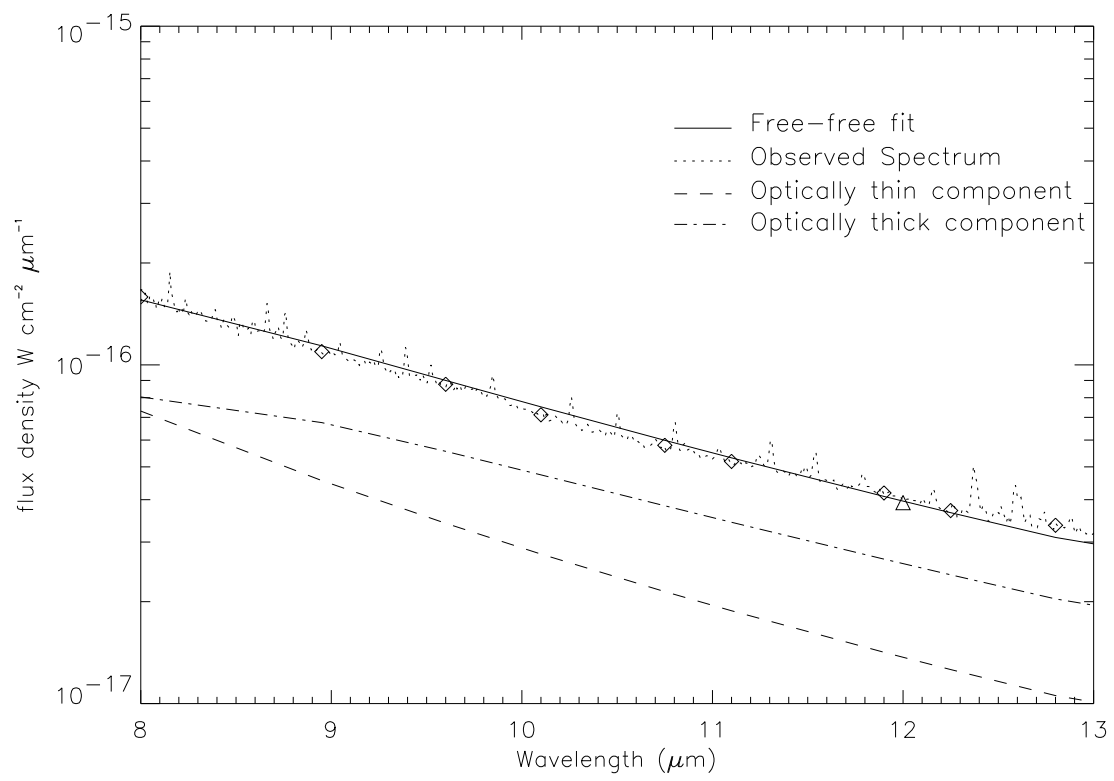

Fig. 4. - The spectrum of $\gamma$ Cas (dotted line), with the free-free emission fit overlaid (solid line). The dashed line and dash-dot line are the optically thin and optically thick components of the emission, respectively. The open diamonds are the points used to make the fit, and the open triangle is the IRAS $12 \mu \mathrm{m}$ flux. 